\title{
Intermittent convective transport carried by propagating electromagnetic filamentary structures in nonuniformly magnetized plasma
}

Xu, G.S.; Naulin, Volker; Fundamenski, W.; Juul Rasmussen, Jens; Nielsen, Anders Henry; Wan, B.N.

Published in:

Physics of Plasmas

Link to article, DOI:

$10.1063 / 1.3302535$

Publication date:

2010

Document Version

Publisher's PDF, also known as Version of record

Link back to DTU Orbit

Citation (APA):

Xu, G. S., Naulin, V., Fundamenski, W., Juul Rasmussen, J., Nielsen, A. H., \& Wan, B. N. (2010). Intermittent convective transport carried by propagating electromagnetic filamentary structures in nonuniformly magnetized plasma. Physics of Plasmas, 17(2), 022501. https://doi.org/10.1063/1.3302535

\section{General rights}

Copyright and moral rights for the publications made accessible in the public portal are retained by the authors and/or other copyright owners and it is a condition of accessing publications that users recognise and abide by the legal requirements associated with these rights.

- Users may download and print one copy of any publication from the public portal for the purpose of private study or research.

- You may not further distribute the material or use it for any profit-making activity or commercial gain

- You may freely distribute the URL identifying the publication in the public portal 


\title{
Intermittent convective transport carried by propagating electromagnetic filamentary structures in nonuniformly magnetized plasma
}

\author{
G. S. Xu, ${ }^{1,2}$ V. Naulin, ${ }^{3}$ W. Fundamenski, ${ }^{1}$ J. Juul Rasmussen, ${ }^{3}$ A. H. Nielsen, ${ }^{3}$ and \\ B. N. Wan ${ }^{2}$ \\ ${ }^{1}$ Euratom-UKAEA, Culham Science Centre, Abingdon OX14 3DB, United Kingdom \\ ${ }^{2}$ Institute of Plasma Physics, Chinese Academy of Sciences, Hefei 230031, China \\ ${ }^{3}$ Association Euratom-Ris $\phi$ DTU, DK-4000 Roskilde, Denmark
}

(Received 11 November 2009; accepted 6 January 2010; published online 9 February 2010)

\begin{abstract}
Drift-Alfvén vortex filaments associated with electromagnetic turbulence were recently identified in reversed field pinch devices. Similar propagating filamentary structures were observed in the Earth magnetosheath, magnetospheric cusp and Saturn's magnetosheath by spacecrafts. The characteristics of these structures closely resemble those of the so-called mesoscale coherent structures, prevailing in fusion plasmas, known as "blobs" and "edge localized mode filaments" in the boundary region, and propagating avalanchelike events in the core region. In this paper the fundamental dynamics of drift-Alfvén vortex filaments in a nonuniformly and strongly magnetized plasma are revisited. We systemize the Lagrangian-invariant-based method. Six Lagrangian invariants are employed to describe structure motion and the resultant convective transport, namely, magnetic flux, background magnetic energy, specific entropy, total energy, magnetic momentum, and angular momentum. The perpendicular vortex motions and the kinetic shear Alfvén waves are coupled through the parallel current and Ampere's law, leading to field line bending. On the timescale of interchange motion $\tau_{\perp}$, a thermal expansion force in the direction of curvature radius of the magnetic field overcomes the resultant force of magnetic tension and push plasma filament to accelerate in the direction of curvature radius resulting from plasma inertial response, reacted to satisfy quasineutrality. During this process the internal energy stored in the background pressure gradient is converted into the kinetic energy of convective motion and the magnetic energy of field line bending through reversible pressure-volume work as a result of the plasma compressibility in an inhomogeneous magnetic field. On the timescale of parallel acoustic response $\tau_{\|} \gg \tau_{\perp}$, part of the filament's energy is transferred into the kinetic energy of parallel flow. On the dissipation timescale $\tau_{d} \gg \tau_{\perp}$, the kinetic energy and magnetic energy are eventually dissipated, which is accompanied by entropy production, and in this process the structure loses its coherence, but it has already traveled a distance in the radial direction. In this way the propagating filamentary structures induce intermittent convective transports of particles, heat, and momentum across the magnetic field. It is suggested that the phenomena of profile consistency, or resilience, and the underlying anomalous pinch effects of particles, heat, and momentum in the fusion plasmas can be interpreted in terms of the ballistic motion of these solitary electromagnetic filamentary structures.
\end{abstract}

[doi:10.1063/1.3302535]

\section{INTRODUCTION}

\section{A. Filamentary structures in fusion plasmas}

Plasma turbulence and the resultant anomalous transport occupied a critical role in the physics of magnetically confined plasmas for thermonuclear fusion research from its very infancy. ${ }^{1}$ Its nonlinearity and complexity are even more prominent in the plasma edge region where the fluctuation levels are typically of the order of unity. ${ }^{2}$ In the last decade accumulating experimental evidences from tokamaks, ${ }^{3}$ stellarators, ${ }^{4}$ reversed field pinches (RFPs), ${ }^{5}$ spherical tokamak, ${ }^{6}$ simple magnetized torus, ${ }^{7}$ and linear devices ${ }^{8}$ revealed the presence of solitary coherent structures ${ }^{9}$ in the plasma turbulence, propagating in the radial direction as well as in the azimuthal direction, commonly referred to as blobs or magnetic-field-aligned filaments, ${ }^{10}$ which lead to intermittent convective transport of particles, heat, momentum, charge, and current across the magnetic field. Recent obser- vations of strong filamentary structures in the plasma boundary in association with edge localized mode (ELM) activity in high confinement mode (H-mode) ${ }^{11-18}$ have many similarities with the blob structures observed in low confinement mode (L-mode), indicating that they might be governed by the same physical mechanisms. By careful analysis of fast camera and reciprocating Langmuir probe data, it was now demonstrated that turbulent transport in the inter-ELM periods is also predominantly carried by filamentary structures, although with much smaller fluctuation amplitudes. ${ }^{6}$

The generation of these structures is generally thought to be the result of strong turbulence at the plasma edge. ${ }^{19-23}$ Their two-dimensional (2D) (in the drift plane, i.e., the plane perpendicular to the magnetic field) electrostatic features have been extensively investigated numerically. ${ }^{20-25}$ Blob related polarization, vorticity, and sheath closure were considered in Refs. 19-23. The interchange dynamics for filament 
motion were highlighted in Refs. 24 and 25. Alfvén closures for 2D blob models and some electromagnetic features were introduced in Refs. 26-29. Transport momentum with blobs was studied in Ref. 30, and kinetic simulation of blob was presented in Ref. 31. The current state of the art in this field was reviewed in Refs. 32 and 33. Now, strong interest is arising around their three-dimensional (3D) characteristics, with strong emphasis on the parallel dynamics ${ }^{34}$ and electromagnetic features. ${ }^{35-37}$ This interest is enhanced by some analogies with the propagating avalanchelike events ${ }^{38}$ or streamers ${ }^{39}$ previously observed in the plasma core region, where the plasma $\beta$ (the ratio of thermal to magnetic pressure) is much higher. These events are generally thought to be associated with some nonlinear electromagnetic structures or fronts ballistically propagating in the radial direction. ${ }^{1}$ Partially motivated by this target, nonlinear kinetic simulations with electromagnetic effects are rapidly evolving in the recent years. ${ }^{40-42}$

The electromagnetic characters of ELM filaments were studied in MAST spherical tokamak ${ }^{14,17}$ and ASDEX Upgrade tokamak ${ }^{15,16}$ experiments, ruling out purely electrostatic dynamics. Hints of electromagnetic features of blobs were obtained in linear devices, ${ }^{43}$ and more recently, the first direct experimental measurement of the parallel current density associated with blob structures ${ }^{44}$ and the first experimental evidence showing the association of the propagating turbulent structures with the drift-Alfvén vortices was obtained in the RFP device. ${ }^{45}$ Also, the first direct observation of current in ELM filaments was made on ASDEX Upgrade tokamak. ${ }^{46}$ These new experimental activities aimed at identifying the electromagnetic features of filamentary structures in the fusion plasmas are partially motivated by recent observation of drift-Alfvén vortices in space plasmas.

\section{B. Filamentary structures in space plasmas}

It is well know that propagating filamentary structures are frequently found at boundary layers in astrophysical, geophysical, and solar atmospheric plasmas. In recent years, dipolar drift-Alfvén vortices have been detected both in the magnetospheric cusp ${ }^{47}$ and in the magnetosheath ${ }^{48-50}$ by the four-spacecraft Cluster mission, and its relation to the crossfield transport was found. ${ }^{51}$ Similar Alfvén vortex filaments were observed in Saturn's magnetosheath by the Cassini spacecraft,${ }^{52}$ indicating the universality of such structures in planetary space. In the year 2007, Alfvén waves were detected in the solar corona, ${ }^{53}$ where the filamentary structures associated with Alfvén waves were suggested to explain how energy is transferred to the solar corona, which is millions of degrees hotter than the solar surface, known as the photosphere. The connection between the erupting filamentary structures in the fusion laboratory plasma and the solar flares was summarized in a recent review paper. ${ }^{54}$ The auroral plasma is occasionally observed to evolve into highly coherent electromagnetic vortex structures, ${ }^{55}$ in which the perturbed electric and magnetic fields exhibit regular rotation together with the particles trapped inside the structures. Another example is the phenomenon that takes place during ionospheric irregularities where localized regions of plasma depletions, often referred to as bubbles, move radially outward on the night side of the equatorial $F$ layer ionosphere. ${ }^{56}$

\section{Impacts of the filamentary transport in fusion plasmas}

The similarity of the electromagnetic filaments in fusion plasmas and in space plasmas suggests that it could be a universal phenomenon in plasma turbulence. As a consequence, the study of plasma blobs or filaments and the resultant intermittent convective transport is one of the most active research areas within plasma physics. ${ }^{2,3,10,33}$ In the fusion plasma boundary the propagating filaments are believed to dominate the transport across the scrapeoff layer (SOL) of fusion devices and possibly lead to serious wall erosion, impurity, and recycling problems for future fusion reactors. ${ }^{33}$ More seriously, the transient power loads associated with the ELM filaments on plasma facing components (both divertor and limit tiles) pose stringer design criteria and operational limits on the material for future fusion reactor, most notably the planned ITER experiment. ${ }^{57}$

An important consequence of the filamentary transport is a strong ballooning nature of turbulence and a strong poloidal asymmetry of radial fluxes in toroidal geometry. Turbulence and flow measurements in the SOL indicate that transport is concentrated on a narrow sector near the outboard midplane, ${ }^{58}$ and the radial fluxes on the outboard midplane are nearly two orders of magnitude larger than on the inboard midplane. ${ }^{59}$ There is also increasingly evidence for the existence a $\mathbf{B} \times \nabla \mathrm{B}$-independent subsonic parallel flow component which is driven by the strong ballooning in the radial turbulent transport, and thus direct from the outboard midplane region toward the divertor targets. ${ }^{60,61}$ These 3D features of turbulent transport have significant implications on ITER, considering that currently ITER design is largely based on predications from 1D transport modeling. ${ }^{57}$ As such, understanding the elementary electromagnetic filamentary structures which constitutes the plasma turbulence is recognized as an issue of the highest priority with regard to ITER.

\section{Lagrangian-invariant-based method}

In this contribution we intend to concentrate our discussions on elementary electromagnetic structures and fundamental mechanism of turbulent transport in the plasma edge. Specifically, the dynamics of drift-Alfvén vortex filaments in a nonuniformly and strongly magnetized plasma are revisited. The physical parameters and derived quantities for a typical filamentary structure [using the Joint European Torus (JET) tokamak parameters] in the pedestal region (in $\mathrm{H}$-mode) or periphery region (in L-mode), in the vicinity of separatrix and in the SOL are summarized in Table I in the Appendix. The Lagrangian-invariant-based method was systemized and intensively used in this paper. Six Lagrangian invariants are employed to describe the structure motion and the resultant convective transport. They are magnetic flux, background magnetic energy, specific entropy, total energy, magnetic momentum, and angular momentum. 
The Lagrangian-invariant-based method was proposed for predicting the quasisteady profiles in tokamak plasmas. ${ }^{62-72}$ The basic assumption is that turbulence mixing causes uniformly distribution of the Lagrangian invariants over the accessible phase space, a state denoted turbulence equipartition (TEP). ${ }^{62}$ The mechanism behind the TEP process is the Rayleigh-Bénard convection, carried by structures, known as the convective cells. ${ }^{73}$ The simplest case of TEP is the adiabatic vertical temperature profile that results from large scale convection in a fluid heated from below. Note that this profile is not determined by the intensity of the turbulence, but by the uniform distribution of specific entropy, which is a Lagrangian invariant.

It is well known that TEP occurs in atmospherical convection, for instance, in the troposphere, and also appears in the convection zone of the sun, a natural nuclear fusion reactor with turbulent transport of energy to the surface, just like in fusion devices. According to data for sun seismology, ${ }^{74}$ the Lagrangian invariant $T n^{-2 / 3}$ is constant to within a factor of $10^{-2}$ in the convection zone. Here $T$ is the temperature and $n$ is the particle density. This is generally attributed to the conservation of the specific entropy, leading to the isentropic atmosphere model. ${ }^{75}$ The same mechanism is responsible for the decrease in temperature with height in the Earth's atmosphere. The success of the TEP approach to turbulent transport in the sun makes it natural to apply the same ideas to fusion devices. ${ }^{62-72}$

Recently, the TEP theory was extensively used to study the anomalous pinch effects of toroidal momentum. ${ }^{76,77}$ In this paper the TEP theory is extended and systemized to include the electromagnetic effects. It will be demonstrated in this paper that the well-know phenomena of profile consistency, or resilience, ${ }^{78}$ and the underlying anomalous pinch (up-gradient transport) effects ${ }^{67}$ of particles, heat, and momentum in fusion plasmas can be interpreted in terms of the ballistic motion of electromagnetic filamentary structures.

\section{E. Paper organization}

This paper is organized as follows. In the following section, we show the relation between the intermittent convective transport and the mesoscale structures using some experimental data from JET tokamak. In Sec. III the ordering scheme for mesoscale structures in fusion plasmas and the concept of drift-Alfvén vortex filaments are introduced. In Sec. IV we highlight that the presence of compressibility in an inhomogeneous magnetic field is responsible for the energy exchange between random thermal motion and collective motion. In Sec. V the generation mechanism of kinetic energy of filament motion and magnetic energy of field line bending is analyzed. The energy transfer is through the reversible pressure-volume work which can be interpreted in terms of the fundamental thermodynamic relation and the entropy equation. The dynamics for filament acceleration and electromagnetic vorticity generation are presented in Sec. VI. An equivalent circuit is used to illustrate the processes in a drift-Alfvén vortex filament. The acceleration of plasma filament is induced by a force unbalance in the direction of curvature radius resulting from plasma inertial response on the timescale of interchange motion, reacted to satisfy quasineutrality. A discussion on the fundamental kinetic mechanism underlying the cross-field turbulent transport associated with the filamentary process is given in Sec. VII. The filamentary structures present a channel for local energy exchange between particles and magnetic field perturbations, leading to breaking of the periodic orbits of particles and the toroidal symmetry of magnetic field and resulting in the violation of the adiabatic invariance associated with the poloidal magnetic flux. In Sec. VIII six Lagrangian invariants are summarized. The filament motion is largely controlled by these Lagrangian invariants. The mode-independent part of the curvature-driven turbulent convective pinch of particles, heat, and toroidal momentum are briefly reviewed in Sec. IX. We employ the quasilinear method to present a qualitative estimation of the intermittent convective transport induced by the radial propagation of the filamentary structures. Finally a discussion of the results is given in Sec. X, followed by a summary. This contribution can be generally regarded as a concept upgrade from electrostatic filamentary structure $^{19-25}$ to electromagnetic filamentary structure $^{26-29,35-37}$ in response to the recent experimental progress $^{43-53}$ in the context of intermittent convective transport mediated by propagating coherent structures. This is a continuation of our previous work presented in Refs. 24, 25, $32,34,54$, and 73 .

\section{INTERMITTENT CONVECTIVE TRANSPORT AND MESOSCALE STRUCTURES}

In this section, we use some reciprocating Langmuir probe data from the JET tokamak to show the relation between the intermittent convective transport and the mesoscale structures. The existence and importance of the mesoscale structures, known as blobs and holes, for cross-field transport in tokamak edge have been demonstrated experimentally, ${ }^{2,3,10}$ where blobs are observed as magneticfield-aligned filaments of enhanced density and temperature as compared with the background plasma, while holes are filaments of reduced density and temperature. Recently, the first experimental evidence showing the formation of blobs and holes in the edge velocity shear layer and the transport of azimuthal momentum by blobs was obtained on JET tokamak. $^{79}$

The most direct indication of turbulence intermittency is reflected by the bursts emerging in the raw signal of the ion saturation current $I_{s} \propto n \sqrt{T_{e}+T_{i}}$, as shown in Fig. 1. For details about this discharge please see Ref. 79. Intermittent positive bursts are prevailing in the SOL, see Fig. 1(a), indicating the presence of blobs. Similar behavior of $I_{s}$ signal has been observed in the SOL of almost all tokamaks, see references in Refs. 2, 3, and 10. At the plasma edge there is a shear layer of poloidal velocity $\Delta r=r-r_{\mathrm{LCFS}}=-15 \sim$ $-3 \mathrm{~mm},{ }^{79}$ where $r_{\mathrm{LCFS}}$ is the minor radius of the last closed flux surface (LCFS). Intermittent negative bursts are detected slightly inside the shear layer, see Fig. 1(c), suggesting the existence of holes. The first report of holes was on DIII-D tokamak. ${ }^{3}$ In the shear layer, positive and negative perturbations are nearly balanced, see Fig. 1(b). It was suggested that 


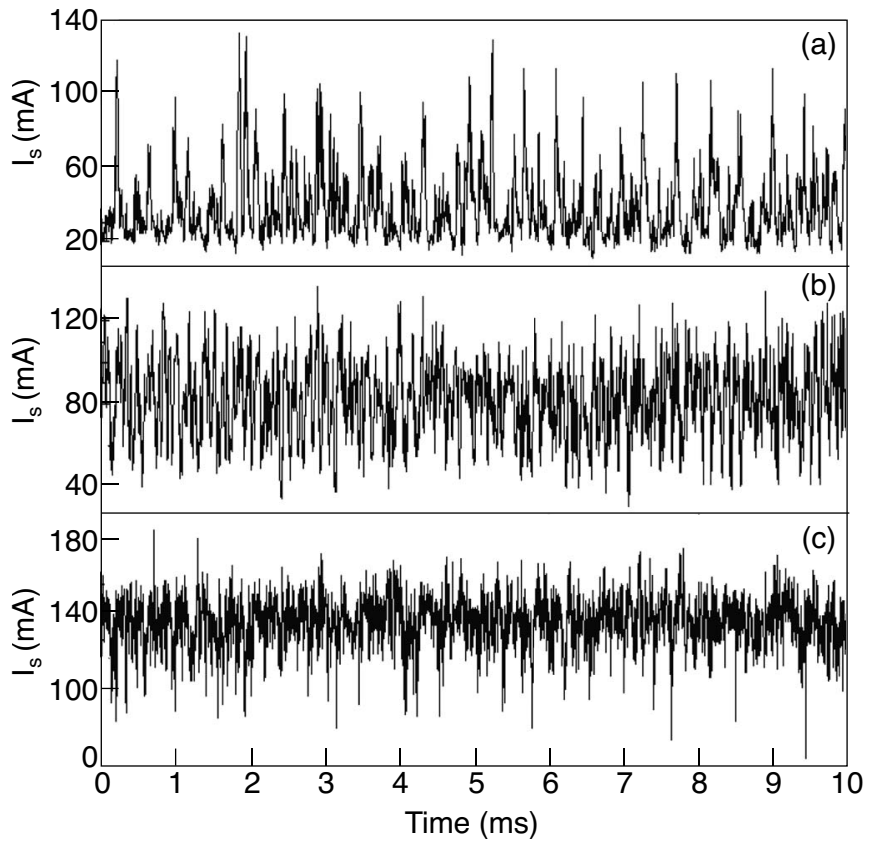

FIG. 1. Turbulence intermittency shown on the raw signal of the ion saturation current $I_{s}$, (a) positive bursts are prevailing in the SOL $\Delta r \sim 28 \mathrm{~mm}$, (b) positive and negative perturbations are nearly balanced in the edge velocity shear layer $\Delta r \sim-10 \mathrm{~mm}$, and (c) negative bursts are prevailing just inside the edge velocity shear layer $\Delta r \sim-18 \mathrm{~mm}$.

the shear layer is the generation region of blobs and holes. ${ }^{79}$

An important feature of turbulence intermittency is the non-Gaussian probability distribution functions (PDFs) of plasma density fluctuations. ${ }^{2,3,10}$ The PDFs of $I_{s}$ fluctuations measured at four radial locations are plotted in Fig. 2. On the horizontal axes, the fluctuation amplitudes have been normalized to the root-mean-square (rms) fluctuation levels of $I_{s}$. In the SOL the PDFs are positively skewed with a heavy tail because of the positive bursts. The skewness $(\mathrm{S})$ and kurtosis $(\mathrm{K})$ of the PDFs, i.e., the deviation of the $I_{s}$ signals from Gaussian statistics, increases from the near SOL to the far SOL, see Figs. 2(a) and 2(b), which was speculated to be due to the reduction in background pressure toward far SOL. ${ }^{79}$ The skewness and kurtosis, defined as the third- and fourth-order moments of the PDF, give a measure of the degree of "asymmetry" and "peakedness" of a distribution with respect to its mean value, respectively. For a Gaussian signal, $S=0$ and $K=3$, whereas for others the deviation from 0 and 3 indicates a higher degree of non-Gaussianity. In the shear layer, the PDF is very close to a Gaussian distribution, as shown in Fig. 2(c). Slightly inside the shear layer, a negative tail appears on the PDF and the skewness changes sign, which can be seen in Fig. 2(d), suggesting the presence of negative bursts.

When many propagating structures with different sizes and velocities pass by probe tips, low-frequency highamplitude fluctuations, constituted by bursts, are detected. Figure 3 shows the power spectra $\ln S\left(k_{\theta}, f\right)$ of floating potential fluctuations, where $k_{\theta}$ is the poloidal wavenumber. The black solid curves show the dispersion relations, which is defined as $\bar{k}_{\theta}(f)=\sum_{k_{\theta}}\left[k_{\theta} S\left(k_{\theta}, f\right) / \sum_{k_{\theta}} S\left(k_{\theta}, f\right)\right]$. Inside the shear layer the turbulence propagates in the electron diamag-

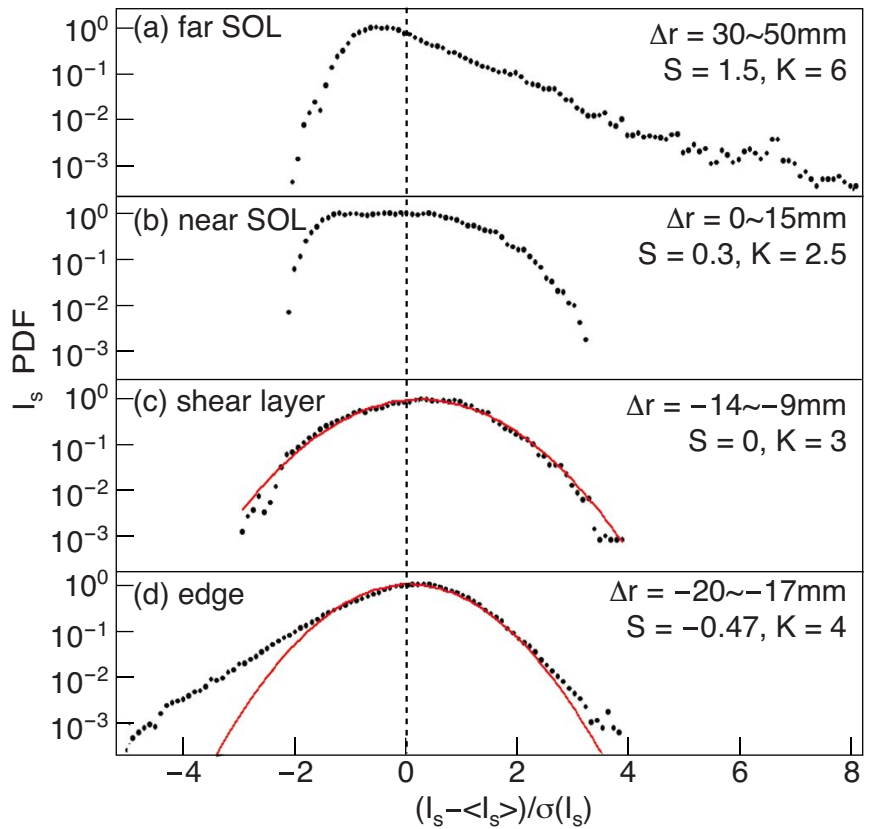

FIG. 2. (Color online) Semilogarithmic plots of the PDFs of the ion saturation current signals $I_{s}$ measured in the (a) far SOL $\Delta r=30-50 \mathrm{~mm}$, (b) near SOL $\Delta r=0-15 \mathrm{~mm}$, (c) edge velocity shear layer $\Delta r=-14$ to $-9 \mathrm{~mm}$, and (d) just inside the edge velocity shear layer $\Delta r=-20$ to $-17 \mathrm{~mm}$. On the horizontal axes, the fluctuation amplitudes have been normalized to the rms fluctuation levels of $I_{s}$. The solid red lines in plotS (c) and (d) are the best Gaussian fit to the PDFs. The corresponding skewness (s) and kurtosis (k) of the PDFs are also shown in the figure.

netic direction as shown in Fig. 3(a), where $\Delta r=-2 \mathrm{~cm}$. Outside the shear layer the turbulence propagates in the ion diamagnetic direction as shown in Fig. 3(b), where $\Delta r$ $=1 \mathrm{~cm}$. The reversion of propagating direction is mainly due

JET Pulse No: 45783

(a) $\Delta_{\mathrm{r}}=-2 \mathrm{~cm}$

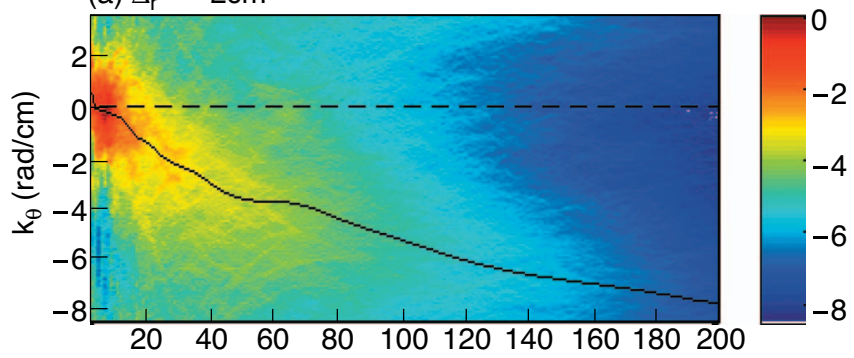

(b) $\Delta_{\mathrm{r}}=1 \mathrm{~cm}$

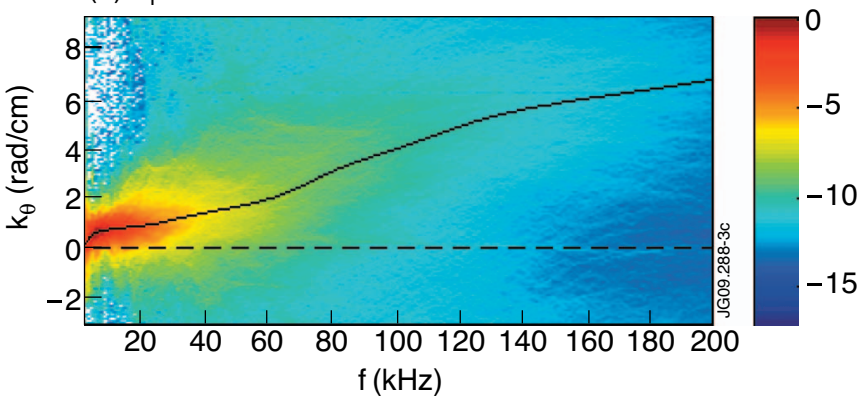

FIG. 3. (Color) Power spectra $\ln S\left(k_{\theta}, f\right)$ of floating potential fluctuations, (a) $2 \mathrm{~cm}$ inside LCFS and (b) $1 \mathrm{~cm}$ outside the LCFS. The spectral intensity is plotted in a logarithmic scale. The black solid curves show the dispersion relations. 


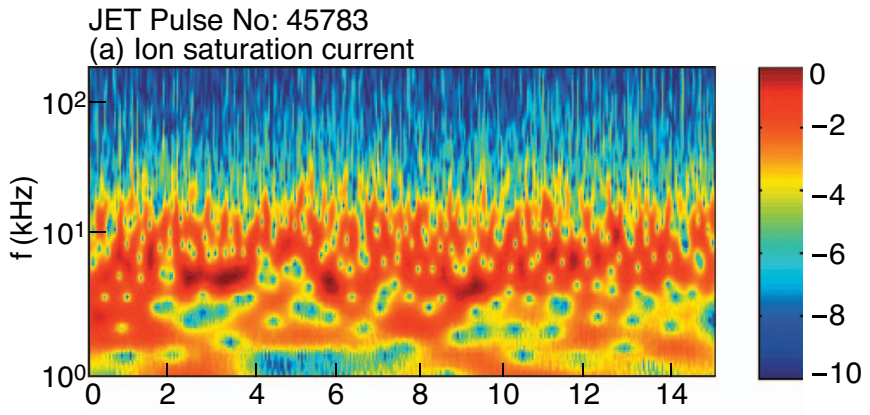

(b) Radial ExB convective velocity

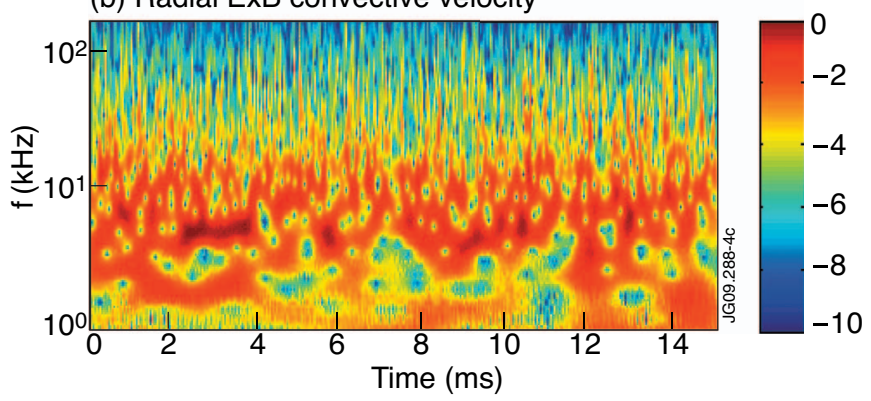

FIG. 4. (Color) Time-frequency wavelet power spectra $\ln S(f, t)$ of (a) ion saturation current $I_{s}$ and (b) radial $\mathbf{E} \times \mathbf{B}$ convective velocity $v_{r}$, in the vicinity of LCFS. The complex Gaussian wavelet and continuous wavelet transform are used to calculate the power spectra. The spectral intensity is plotted in a logarithmic scale.

to the radial variation of the $\mathbf{E}_{\mathbf{r}} \times \mathbf{B}$ rotation velocity. ${ }^{79}$ From Fig. 3 one can see that most of the spectrum power distributes in the low-frequency low-wavenumber regions $(|f|$ $<40 \mathrm{kHz}$ and $\left|k_{\theta}\right|<4 \mathrm{rad} / \mathrm{cm}$ ), which has been demonstrated to be in association with the mesoscale structures. ${ }^{79}$

The nature of turbulence intermittency can be further characterized by the time behavior of the power spectra, for which the complex Gaussian wavelet and continuous wavelet transform are used. Figure 4 shows the time-resolved wavelet power spectra $\ln S(f, t)$ of (a) ion saturation current $I_{s}$ and (b) radial $\mathbf{E} \times \mathbf{B}$ convective velocity $v_{r}$, in the vicinity of LCFS. Consistent with Fig. 3, the spectrum power concentrates in the low-frequency region. Some intermittent structures can be identified around $10 \mathrm{kHz}$, possibly with some overlapping of adjacent structures. Comparing Figs. 4(a) and 4(b), one can find the structures in the $I_{s}$ signal and the $v_{r}$ signal are strongly correlated, indicating that these structures are propagating in the radial direction driven by the $\mathbf{E} \times \mathbf{B}$ drift, suggesting the interchange drive as the underlying mechanism governing structure motion, as depicted in Refs. 24 and 25. The correlation was further confirmed by the conditional average analysis, as shown in Fig. 5 of Ref. 79, indicating that the fluctuations of the $I_{s}$ and the $v_{r}$ tend to be in phase, i.e., charge polarization is nearly at the center of the structures, which maximizes the convective transport.

The cross-field convective transport is dominantly carried by these propagating structures. Figure 5 shows the wavenumber power spectra $S\left(k_{\theta} \rho_{s}\right)$ of density fluctuation $\tilde{n}$, poloidal electric field fluctuation $\widetilde{E}_{\theta}$, and radial convective particle flux $\Gamma=\left\langle\widetilde{n} \widetilde{E}_{\theta}\right\rangle / B_{0}$, measured in the vicinity of LCFS, where $\rho_{s}$ is the ion sound gyroradius and at this location $\rho_{s}$

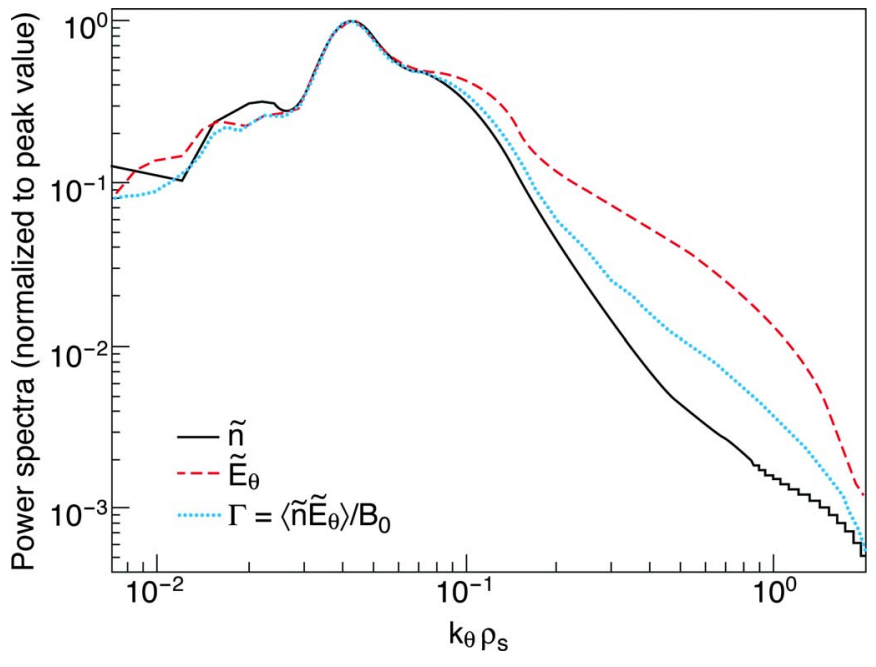

FIG. 5. (Color online) Wavenumber power spectra $S\left(k_{\theta} \rho_{s}\right)$ of density fluctuation $\widetilde{n}$ (solid black line), poloidal electric field fluctuation $\widetilde{E}_{\theta}$ (dashed red line) and radial convective particle flux $\Gamma=\left\langle\widetilde{n} \widetilde{E}_{\theta}\right\rangle / B_{0}$ (dotted blue line), in the vicinity of LCFS.

$\approx 0.54 \mathrm{~mm}$. From the figure one can see that the spectrum power concentrates in the low-wavenumber region with $k_{\theta} \rho_{s} \ll 1$, implying that the structure size is much bigger than $\rho_{s}$, whereas, compared with the system size $a$, i.e., the minor radius of plasma, these structures are still small, therefore they are usually called mesoscale coherent structure (versus smallscale turbulence). ${ }^{2,3,10,33}$ The radial convective particle flux is dominated by these structures, as indicated in Fig. 5. We use these data as a brief introduction so that one can have a direct idea for what are mesoscale structures in fusion plasma edge and why we want to study these structures.

\section{PHYSICS BEHIND THE MESOSCALE STRUCTURES}

From the theoretical point of view it has been shown that the edge turbulence is electromagnetic even for low local value of plasma $\beta .^{80-82}$ Fully electromagnetic nonlinear gyrokinetic theory for edge turbulence has now come to be mature. ${ }^{83,84}$ Efforts dedicated to the development of kinetic simulation of edge turbulence are now underway. ${ }^{85}$ Concerning the basic mechanism, in the recent years substantial progress has been made in understanding the radial propagation of blobs in the plasma boundary. ${ }^{19-33,86-89}$ For toroidally magnetized plasmas this was suggested to be due to the guiding center drifts caused by an inhomogeneous magnetic field, resulting in a vertical charge polarization ${ }^{90}$ and a resultant $\mathbf{E} \times \mathbf{B}$ radial convection reacted to satisfy quasineutrality, $\nabla \cdot \mathbf{j}=0 .{ }^{19-25}$

\section{A. Quasineutrality}

Employing the quasineutral condition implies $c / v_{A}$ $=\omega_{p i} / \omega_{c i} \sim \rho_{s} / \lambda_{D} \gg 1$, where $c$ is the speed of light, $v_{A}$ $\equiv\left(B_{0}^{2} / \mu_{0} m_{i} n\right)^{1 / 2}$ is the Alfvén speed, $\omega_{p i} \equiv\left(n e^{2} / m_{i} \varepsilon_{0}\right)^{1 / 2}$ is the ion plasma frequency, $\omega_{c i} \equiv e B_{0} / m_{i}$ is the ion gyrofrequency, $c_{s} \equiv\left[\left(T_{i}+T_{e}\right) / m_{i}\right]^{1 / 2} \sim \beta_{e}^{1 / 2} v_{A}$ is the ion sound speed, $\rho_{s} \equiv c_{s} / \omega_{c i}$ is the ion sound gyroradius, $\lambda_{D} \equiv\left(\varepsilon_{0} T_{e} / n e^{2}\right)^{1 / 2}$ 
$\sim c_{s} / \omega_{p i}$ is the Debye length, $\beta_{e} \equiv 2 \mu_{0} p_{e} B_{0}^{-2} \sim\left(c_{s} / v_{A}\right)^{2}$ $=\left(\rho_{s} / L_{p i}\right)^{2}$ is the ratio of electron pressure to magnetic pressure, and $L_{p i} \equiv c / \omega_{p i}$ is the ion inertial skin length. The permittivity in a quasineutral plasma is $\varepsilon=\left(\rho B_{0}^{-2}+\varepsilon_{0}\right) \approx \rho B_{0}^{-2}$ and the permeability in a low- $\beta$ plasma is $\mu=\mu_{0}+\mu_{0}\left(\mathbf{B} / \mu_{0} \mathbf{M}\right.$ $-1)^{-1} \approx \mu_{0}$, where $\rho \equiv m_{i} n$ is the mass density (note $\rho_{i}$ is the ion gyroradius) and $\mathbf{M} \equiv-B^{-1} p_{\perp} \mathbf{b}$ is the magnetization. With the permittivity and the permeability, the Alfvén speed is formally defined as $v_{A} \equiv(\mu \varepsilon)^{-1 / 2}$. Assuming singly charged ions, as herein, $n$ refers to both the electron or ion density. In fusion plasmas, even in the SOL, the quasineutral condition is well satisfied, please see Table I.

\section{B. Ordering scheme for mesoscale structures}

The typical cross-field size $L_{\perp}$ of the filamentary structures was observed to be close to the ion poloidal gyroradius and the ion inertial length $L_{\perp} \sim \rho_{\theta i} \sim L_{p i} \gg \rho_{s}$, ,3 which does not follow the standard gyrokinetic ordering of drift-wave microturbulence $k_{\perp} \rho_{s} \sim 1,{ }^{42,84}$ thereby they are usually called mesoscale coherent structures, where $k_{\perp}$ is the perpendicular wavenumber, $\rho_{\theta i} \equiv \rho_{i} B_{0} / B_{\theta}$ is the ion poloidal gyroradius, $\rho_{i} \equiv v_{t h i} / \omega_{c i}$ is the ion gyroradius, and $v_{t h i} \equiv\left(2 T_{i} / m_{i}\right)^{1 / 2}$ is the ion thermal velocity.

The ion inertial length $L_{p i}$ is typically of the same order as the radial gradient lengths of background pressure $\langle p\rangle$ and zonal potential $\langle\phi\rangle, L_{p i} \sim L_{p} \sim L_{\phi}$ in the plasma edge of tokamaks, where usually steep pressure gradient and strong radial electric field present, please see Table I, here $L_{p}$ $\equiv\left(\partial_{r} \ln \langle p\rangle\right)^{-1}, L_{\phi} \equiv\left(\partial_{r} \ln \langle\phi\rangle\right)^{-1}$, and $\langle\cdots\rangle$ denotes average over a flux surface, i.e., the surface with constant poloidal magnetic flux $\psi_{p}$. These structures are strongly elongated along the field lines, ${ }^{2}$ manifested as magnetic-field-aligned filaments. In tokamak geometry the typical parallel scale length of a filament $L_{\|}$is of the same order of the parallel geometry length $\pi q R$, where $q$ is the magnetic safety factor and $R$ is the major radius.

Following Refs. 54 and 91, we employ the drift ordering for small parallel gradient. The magnetization parameter $\delta$ $\equiv \rho_{s} / L_{\perp} \ll 1$ is used as a measure of the smallness of the $\mathbf{E}$ $\times \mathbf{B}$ drift velocity $u_{E} / c_{s} \sim \delta$, where $\mathbf{u}_{E} \equiv B^{-2} \mathbf{E} \times \mathbf{B}$. By this ordering the vortex turnover time $\tau_{\perp} \equiv L_{\perp} / u_{E} \sim \delta^{-1} \tau_{s}$ $\sim \delta^{-2} \tau_{c i}$ is much longer than the ion gyroperiod $\tau_{c i} \equiv \omega_{c i}^{-1}$, therefore only low-frequency dynamics are involved, where $\tau_{s} \equiv L_{\perp} / c_{s}$ is the perpendicular ion sound transit time. ${ }^{36} \mathrm{In}$ this paper $\tau_{\perp}$ is referred to the timescale for the interchange (or convective) motion. The ratio between poloidal and total magnetic fields in a tokamak is $\vartheta \equiv B_{\theta} / B_{0}=\in / q=\rho_{i} / \rho_{\theta i}$ $\sim \delta$, where $\in \equiv r / R \ll 1$ is the local inverse aspect ratio.

The ion guiding center drift velocity is composed of $\mathbf{u}$ $=\mathbf{u}_{\|}+\mathbf{u}_{E}+\mathbf{u}_{G i}+\mathbf{u}_{p}+\mathbf{u}_{\mathrm{FLR}}$, where $\mathbf{u}_{\|} \equiv u_{\|} \mathbf{b}$ is the parallel flow velocity, $\mathbf{b} \equiv \mathbf{B} / B$ is the unit magnetic field vector, $\mathbf{u}_{G i}$ $=e^{-1} B^{-1}\left(T_{i \perp} \mathbf{b} \times \nabla \ln B+T_{i \|} \nabla \times \mathbf{b}\right)$ is the curvature and grad-B drift velocities, $\mathbf{u}_{p} \equiv B^{-1} \tau_{c i} d_{t} \mathbf{E}_{\perp}$ is the ion polarization drift velocity, the total time derivative is given by $d_{t}$ $\equiv \partial_{t}+\mathbf{u} \cdot \nabla$, and $\mathbf{u}_{\mathrm{FLR}}=(1 / 4) \rho_{i}^{2} \nabla_{\perp}^{2} \mathbf{u}_{E}$ is due to the finiteLarmor-radius (FLR) effect. By this ordering we have $u_{G i} / u_{E} \sim \rho_{i} v_{t h i} / R u_{E} \sim L_{\perp} / R \sim \delta, \quad u_{p} / u_{E} \sim \tau_{c i} / \tau_{\perp} \sim \delta^{2}, \quad$ and $u_{\mathrm{FLR}} / u_{E} \sim \rho_{i}^{2} / L_{\perp}^{2} \sim \delta^{2}$. Consequently, the $\mathbf{E} \times \mathbf{B}$ drift is the only lowest order cross-field guiding center drift, which is moreover the same for both positively and negatively charged particles. To lowest order, the ion guiding center drift velocity is reduced to $\mathbf{u} \approx \mathbf{u}_{\|}+\mathbf{u}_{E}$.

\section{In the finite- $\boldsymbol{\beta}$ plasmas}

We only consider the case of strongly magnetized plasma, where the plasma $\beta$ is low, $\beta_{e} \sim\left(c_{s} / v_{A}\right)^{2}=\left(\rho_{s} / L_{p i}\right)^{2}$ $\sim \delta^{2}$. For the case of greatest current interest, $\beta_{e}>m_{e} / m_{i}$, i.e., in a finite- $\beta$ plasma, ${ }^{42}$ the electromagnetic effects are important. In this case the Alfvén speed $v_{A}$ is smaller than the electron thermal velocity $v_{\text {the }} \equiv\left(2 T_{e} / m_{e}\right)^{1 / 2}$, i.e., $v_{A} / v_{\text {the }}$ $\sim\left(m_{e} / m_{i} \beta_{e}\right)^{1 / 2}<1$. From Table I one can see this is generally the case inside the separatrix, i.e., in the closed field line region. In the vicinity of the separatrix, we have $m_{e} / m_{i}$ $\sim \beta_{e} \sim \delta^{2}$ and hence $\rho_{e} / \rho_{i}=v_{t h i} / v_{\text {the }} \sim L_{p e} / L_{p i} \sim\left(m_{e} / m_{i}\right)^{1 / 2}$ $\sim \delta$, where $L_{p e} \equiv c / \omega_{p e}$ is the electron inertial skin length and $\omega_{p e} \equiv\left(n e^{2} / m_{e} \varepsilon_{0}\right)^{1 / 2}$ is the electron plasma frequency.

At fusion plasma edge, the vortex turnover time $\tau_{\perp}$ is comparable with the shear Alfvén time $\tau_{A} \equiv L_{\|} / v_{A}$ (see Table I), so that the electrostatic vortex motions and the kinetic shear Alfvén waves (KSAWs) are coupled, $\tau_{\perp} \sim \tau_{A}$. The coupling with the compressional Alfvén wave, i.e., the magnetosonic wave, is negligible, since $\tau_{\perp}$ is much longer than the compressional Alfvén time $\tau_{A \perp} \equiv L_{\perp} / v_{A} \sim \delta \tau_{s} \sim \delta^{2} \tau_{\perp} \sim \tau_{c i}$, where $c_{s} / v_{A} \sim \beta_{e}^{1 / 2} \sim \delta$ has been used. By this ordering the degree of the spatial anisotropy is $L_{\perp} / L_{\|} \sim u_{E} / v_{A} \sim \delta \beta_{e}^{1 / 2}$ $\sim \delta^{2}$. Using the quasineutral condition $\nabla \cdot \mathbf{j}=0$, we have $j_{\perp} / j_{\|} \sim B_{\|} / B_{\perp} \sim A_{\perp} / A_{\|} \sim L_{\perp} / L_{\|} \sim \delta^{2}$, where $B_{\|}$is the parallel magnetic perturbation, and $A_{\perp}$ is the perpendicular component of the magnetic vector potential. $B_{\|}$and $A_{\perp}$ are neglected in this paper since they are associated with the compressional Alfvén wave. The timescale for parallel acoustic response is $\tau_{\|} \equiv L_{\|} / c_{s} \sim \delta^{-1} \tau_{\perp}$, indicating that the excitation of the parallel ion sound wave is a slow process compared with the perpendicular convective motion. This explains why $2 \mathrm{D}$ approximation is frequently applied to the ion motion and electrostatic vortex dynamics.

When the filaments and the KSAW are coupled, the parallel phase speed $v_{\mathrm{ph} \|} \equiv \omega / k_{\|}=L_{\|} / \tau_{\perp}$ is of the order of $v_{A}(1$ $\left.+k_{\perp}^{2} \rho_{s}^{2}\right)^{1 / 2}$. The relative wave impedance $\varsigma \equiv u_{F} / u_{E}=v_{\mathrm{ph} /} / Z$ depends not only on the plasma $\beta$ but also on the scale $k_{\perp} \rho_{s},{ }^{92,93}$ where $\mathbf{u}_{F}$ is the perpendicular velocity of field lines, $Z \equiv E_{\perp} / B_{\perp}=u_{E} B_{0} / B_{\perp} \sim \phi / A_{\|}$is the wave impedance, here $\phi$ is the scalar potential, and $A_{\|}$is the parallel component of the magnetic vector potential. In a finite- $\beta$ plasma (from Table I one can see this is generally the case inside the separatrix), for mesoscale structures $\left(k_{\perp} \rho_{s} \ll 1\right), v_{\text {phl }} \sim v_{A}$, the wave impedance $Z$ is of the same order of the Alfvén speed $v_{A}$ and structures move perpendicularly with a velocity $u_{E}$ $\sim \delta c_{s}$, whereas the wave impedance $Z$ of smallscale structures $\left(k_{\perp} \rho_{s} \sim 1\right)$ substantially exceed $v_{A}$; they move with small velocities $u_{E} \ll \delta c_{s}$ and are strongly coupled with drift waves. Note, for the smallscale structures, one should apply the standard gyrokinetic ordering instead of the ordering scheme for mesoscale structures.

In the ideal magnetohydrodynamic (MHD) limit, $s=1$, the field lines are exactly frozen in fluid elements and mov- 
ing with them. In the electrostatic limit, $\varsigma \ll 1$. For mesoscale structures, $\varsigma$ is of order unity, ${ }^{92,93}$ implying that the electromagnetic effects are more important for the mesoscale dynamics than the smallscale dynamics. This gives the ordering of the perpendicular magnetic field fluctuations $B_{\perp} / B_{0}$ $=u_{F} / v_{\mathrm{ph} \|} \sim \mathrm{s} \delta \beta_{e}^{1 / 2} \sim \mathrm{s} \delta^{2}$, which is typically very small due to the low $\beta$. But, the magnetic fluctuation levels increase with plasma $\beta$, so that field line bending in high- $\beta$ plasmas is generally stronger than in low- $\beta$ plasmas. Recent experiments on blobs from a RFP device ${ }^{44,45}$ and ELM filaments from ASDEX Upgrade tokamak ${ }^{46}$ suggested some MHD behaviors, $s \sim 1$.

The parallel wavelength of the shear Alfvén wave $L_{\|}$ $=v_{A} \tau_{A}$ is comparable with the typical parallel scale length $\pi q R$ in a tokamak, implying that the induced field line bending is global in the parallel direction, ${ }^{33}$ but is localized in the drift plane with the cross-field size $L_{\perp}$, manifested in a tokamak by a finite toroidal mode number. The coupling with KSAW allows a perpendicular displacement of the field lines by an amount of $r_{\perp} \equiv L_{\|} B_{\perp} / B_{0}=u_{F} \tau_{A} \sim \varsigma L_{\perp}$, which defines the radius of an Alfvén vortex. Inside an Alfvén vortex the perturbed electric and magnetic fields are coupled with each other through Maxwell's equations and exhibit regular rotation together with the particles trapped inside the structures. ${ }^{9}$ In such a way the plasma could evolve into highly coherent electromagnetic vortex structures.

The electrostatic fluctuation levels are of order unity at the plasma edge, $e \phi / T_{e} \sim \widetilde{n} /\langle n\rangle \sim \widetilde{p}_{e} /\left\langle p_{e}\right\rangle \sim 1$, where $\widetilde{n} \equiv n$ $-\langle n\rangle, \widetilde{p}_{e} \equiv p_{e}-\left\langle p_{e}\right\rangle$, and $\widetilde{\phi} \equiv \phi-\langle\phi\rangle$. Here, $\langle\phi\rangle$ is the zonally averaged potential. The electrostatic fluctuation levels are consistent with the ordering, $e \phi / T_{e} \sim u_{E} / c_{s} \delta \sim 1$.

\section{Alfvén vortex filaments}

The strong mobility of the electrons along the field lines allows a parallel current $\mathbf{j}_{\|}$to arise as a response to the charge polarization induced by curvature and grad-B drifts in the drift plane. ${ }^{24,25}$ This current provides a channel to couple the electrostatic vortex dynamics with the KSAW. ${ }^{44-52}$ The coupling is through Ampere's law $\mu_{0} \mathbf{j}_{\|}=\nabla \times \mathbf{B}_{\perp}=-\mathbf{b} \nabla_{\perp}^{2} A_{\|}$, where the total magnetic field is $\mathbf{B}=\mathbf{B}_{0}+\mathbf{B}_{\perp}, \mathbf{B}_{0}$ is the background (static equilibrium) magnetic field, $\hat{\mathbf{z}} \equiv \mathbf{B}_{0} / B_{0}$ is the unit vector along $\mathbf{B}_{0}, \mathbf{B}_{\perp} \equiv \nabla \times \mathbf{A}_{\|}=-\hat{\mathbf{z}} \times \nabla A_{\|}$is the perpendicular (to $\mathbf{B}_{0}$ ) magnetic perturbation, with $B^{2}=B_{0}^{2}+B_{\perp}^{2}$ and $\nabla \cdot \mathbf{B}=\nabla \cdot \mathbf{B}_{0}=\nabla \cdot \mathbf{B}_{\perp}=0$.

These more general vortices are denoted Alfvén vortex filaments ${ }^{94-96}$ and are characterized by both an electrostatic vorticity $\boldsymbol{\Omega} \equiv \nabla \times \mathbf{u}_{E}=\mathbf{b} B^{-1} \nabla_{\perp}^{2} \phi$ and a magnetic vorticity $\boldsymbol{\Omega}_{A} \equiv \mu_{0} \mathbf{j}_{\|}$, where $\mathbf{u}_{E} \equiv B^{-2} \mathbf{E} \times \mathbf{B} \approx B_{0}^{-1} \hat{\mathbf{z}} \times \nabla \phi$ and the electric field is $\mathbf{E} \equiv-\nabla \phi-\partial_{t} \mathbf{A}_{\|}$. The perpendicular electric field is nearly electrostatic $\mathbf{E}_{\perp}=-\nabla_{\perp} \phi$, while the parallel electric field involves magnetic perturbation through $E_{\|}=-\partial_{\|} \phi-\partial_{t} A_{\|}$ $=\eta_{\|} j_{\|}-\partial_{\|} \Psi$, where $\Psi \equiv \int\left(\eta_{\|} j_{\|}-E_{\|}\right) d l_{\|}$is the parallel electromotive force (emf); here we defined $l_{\|}$as the distance along the perturbed field line, i.e., $\partial_{\|} \equiv \partial / \partial l_{\|}=\mathbf{b} \cdot \nabla=\partial_{z}+B_{0}^{-1} \nabla A_{\|}$ $\times \hat{\mathbf{z}} \cdot \nabla, \partial_{t} \equiv \partial / \partial t$, and $\partial_{z} \equiv \hat{\mathbf{z}} \cdot \nabla$. The parallel derivatives carry nonlinearity entering through $A_{\|}$. Then, the parallel potential gradient and the parallel electric field can be written as $\partial_{\|} \phi$ $=\partial_{z} \phi+\mathbf{u}_{E} \cdot \nabla A_{\|}$and $E_{\|}=-\partial_{z} \phi-\hat{d}_{t} A_{\|}$, where $\hat{d}_{t} \equiv \partial_{t}+\mathbf{u}_{E} \cdot \nabla$ is the transverse advective derivative.

When an Alfvén vortex arises in a plasma with strong background pressure gradient, which is a typical case in fusion plasmas, it will propagate in the azimuthal direction and couple to the drift wave, so that it is usually called driftAlfvén vortex. ${ }^{97}$ The concept of drift-Alfvén vortex was recently applied to the space plasma to interpret the Cluster observations in the Earth's magnetosphere. ${ }^{92,93}$

\section{E. Ordering in generalized Ohm's law}

In the vicinity of the separatrix, the inertial parameter $\hat{\boldsymbol{\varpi}}=m_{e} / m_{i}\left(q R / L_{p}\right)^{2}>1$, the inductive parameter $\hat{\beta}$ $=\beta_{e}\left(q R / L_{p}\right)^{2}>1$, and the resistive parameter $C$ $=0.51 \hat{\varpi} L_{p} / c_{s} \tau_{e i}>1$ are of the same order (see Table I), ${ }^{36}$ which implies strong nonadiabatic electron activity due to the inertial, inductive, and resistive parallel electron responses. With $q R / v_{\text {the }}, L_{p} / c_{s}$, and $\tau_{e i}$ all comparable (see Table I), the situation is referred to as transcollisional, where $\tau_{e i} \propto T_{e}^{3 / 2} n^{-1}$ is the electron-ion collisional time.

The parallel component of generalized Ohm's law is given by ${ }^{42}$

$$
\begin{aligned}
\partial_{t} A_{\|} & +\eta_{\|} j_{\|}+e^{-2} m_{e} \hat{d}_{t}\left(j_{\|} n^{-1}\right) \\
& =e^{-1} n^{-1}\left[\partial_{\|} p_{e \|}-\left(p_{e \|}-p_{e \perp}\right) \partial_{\|} \ln B\right]-\partial_{\|} \phi,
\end{aligned}
$$

where $\eta_{\|}$is the parallel resistivity and it is about two times smaller than the perpendicular resistivity $\eta_{\perp}=1.96 \eta_{\|}$ $=m_{e} /\left(n e^{2} \tau_{e i}\right), p_{e \|} \equiv n T_{e \|}$ is the electron parallel pressure and $p_{e \perp} \equiv n T_{e \perp}$ is the electron perpendicular pressure. Equation (1) is also known as the electron parallel force balance equation. With the parallel emf, it can be written as $\partial_{t} A_{\|}+\eta_{\|} j_{\|}$ $=\partial_{\|}(\Psi-\phi)$. The first term on the left-hand side (LHS) and the two terms on the right-hand side (RHS) of Eq. (1) are of the same order. Using $\partial_{\|} \phi$ as a reference, we have the ordering of the first terms on the LHS $\sim \tau_{A} / \tau_{\perp} \sim 1$ and the first term on the RHS $\sim \widetilde{p}_{e} T_{e} / p_{e} e \phi \sim 1$. The second term (the Joule dissipation term $\left.\sim \tau_{A} L_{p e}^{2} / \tau_{e i} L_{\perp}^{2} \sim m_{e} \tau_{\perp} / m_{i} \tau_{e}\right)$ and the third term (the electron inertial term $\sim \tau_{A} L_{p e}^{2} / \tau_{\perp} L_{\perp}^{2} \sim m_{e} / m_{i}$ ) on the LHS are small compared to the other terms by a factor of $m_{e} / m_{i}$.

From Table I one can see that in the whole boundary region $\tau_{\perp}>\tau_{e i}$, indicating that the Joule dissipation term dominates over the electron inertial term. Only in the nearly collisionless plasma core region where $\tau_{\perp}<\tau_{e i}$, the electron inertial term is more important. For simplifying the discussion, the electron inertia and the electron viscosity are omitted in this paper, since we are mainly interested in the plasma edge region, otherwise the electron kinetic energy must be taken into account in the energy conservation.

From the above ordering analysis we find the first term on the LHS, i.e., the magnetic induction terms, is of the same order of the terms on the RHS, indicating that the magnetic induction effects are important for mesoscale dynamics. The physics reflected by Eq. (1) is commonly interpreted as the response of the parallel current $j_{\|}$to the net parallel gradient force $e n \partial_{\|}(\Psi-\phi)$ on electrons. ${ }^{36}$ Any force imbalance represented by a nonvanishing RHS will excite a $j_{\|}$and the result- 
ant magnetic fluttering $A_{\|}$. When this force is zero, the two gradients balance and the electrons are said to be "adiabatic." Here, the adiabatic means these electrons do not exchange energy with the magnetic field. The "adiabatic electrons" are expected to follow the Boltzmann distribution $\tilde{n} /\langle n\rangle$ $=e \widetilde{\phi} /\left\langle T_{e}\right\rangle .{ }^{98}$ When all the electrons are adiabatic electrons, the drift waves are in the electrostatic limit. The parallel phase velocity of a drift wave is expected to be in the range $v_{\text {thi }}<v_{\text {ph\|l }}<v_{\text {the }}$.

In the SOL the electron temperature is so low that usually $v_{A}>v_{\text {the }}$ and $\tau_{\|}^{e}>\tau_{\perp}$, where $\tau_{\|}^{e} \equiv L_{\|}^{2} / \chi_{\|}^{e}$ is the electron parallel thermal conductive time and $\chi_{\|}^{e} \equiv 3.2 \tau_{e i} T_{e} / m_{e}$ is the electron parallel thermal conductivity (see Table I), implying that the mobility of electrons along field lines is relatively weak. The drift-wave phase velocity condition $v_{\text {phll }}<v_{\text {the }}$ is unsatisfied if $v_{\mathrm{ph} \|} \sim v_{A}$, thereby for mesoscale structures the coupling to drift wave could be weak and there could be a significant number of "nonadiabatic electrons." This explains why the interchange turbulence is prevailing in the SOL (Ref. 34) and the SOL turbulence is electromagnetic even for low local value of plasma $\beta$. $^{37}$

Inside the separatrix, although $v_{\mathrm{ph} \|}<v_{\text {the }}$ is generally satisfied and $\tau_{\|}^{e}<\tau_{\perp}$, the electron temperature isotropization time $\tau_{T e}$ becomes comparable with or even longer than $\tau_{\perp}$ (see Table I), so that the trapped electron effects become important. The trapped electrons do not obey the Boltzmann relation and they generally contribute to the so-called nonadiabatic response. Therefore, it could be everywhere (from the SOL to the plasma core) that a significant percentage of nonadiabatic electrons exists and the turbulence is electromagnetic in nature.

When the RHS of Eq. (1) does not vanish, reflecting an imbalance in the parallel force on electrons, the parallel current $j_{\|}$and magnetic perturbations $A_{\|}$will arise as a result of the magnetic induction, driven by the so-called nonadiabatic part of the density fluctuations $\tilde{h} \equiv \widetilde{n}-e \widetilde{\phi}\langle n\rangle /\left\langle T_{e}\right\rangle{ }^{36}$ It is called nonadiabatic, because during this process the energy of particle system is not conserved. This nonadiabatic part of density fluctuations provides a channel to exchange internal energy of particles with the magnetic energy of field line bending. The second term on the LHS of Eq. (1), i.e., the Joule dissipation term, is responsible for the irreversible magnetic energy dissipation, governing the magnetic diffusion process. It is irreversible since it is accompanied by entropy production.

\section{F. Alfvén's frozen-in law and magnetic diffusion}

In the ideal MHD limit ( $s=1$ ), Alfvén's frozen-in law is an accurate law: the field lines are exactly frozen in fluid elements and moving with them, and the parallel emf $\Psi$ vanishes. In the resistive MHD case $(\mathrm{s}<1)$, only collisional resistivity can break Alfvén's frozen-in law. When kinetic effects, such as Landau damping and/or trapped particles, ${ }^{42}$ are taken into account $(\varsigma<1)$, Alfvén's frozen-in law can also be broken by the kinetic effects and generating the parallel emf $\Psi$ (note in this paper the definition of the parallel emf excludes the contribution from the collisional resistivity). The kinetic effects are alternatives to resistive diffusion for decoupling the magnetic field and plasma. In collisionless plasmas the former dominates over the latter. The presence of the parallel emf and/or collisional resistivity allows plasma to drift across the field lines.

With the help of generalized Ohm's law (1), the electric field can be expressed as

$$
\mathbf{E}=-\nabla_{\perp} \phi-\nabla_{\|} \Psi+\eta \mathbf{j} .
$$

Note $\eta_{\perp} j_{\perp} / \eta_{\|} j_{\|} \sim L_{\perp} / L_{\|} \sim \delta^{2}$. Taking the curl of the electric field expression, we obtain the induction equation, i.e., the differential form of Alfvén's frozen-in law,

$$
\partial_{t} \mathbf{B}_{\perp}=\nabla \times\left(\mathbf{u}_{E} \times \mathbf{B}\right)+\nabla \times \nabla_{\|} \Psi+D_{m} \nabla^{2} \mathbf{B}_{\perp}-\nabla \eta \times \mathbf{j},
$$

where $D_{m} \equiv \mu_{0}^{-1} \eta \propto T_{e}^{3 / 2}$ is the magnetic diffusivity. The first term on the RHS of Eq. (3) can be written as $-\nabla \times \nabla_{\|} \phi$. The LHS and the first term (frozen term) and the second term (drift term) on the RHS are of the same order. The third term (magnetic diffusion term) and the last term (resistance gradient term) $\sim \tau_{\perp} L_{p e}^{2} / \tau_{e i} L_{\perp}^{2} \sim \tau_{\perp} m_{e} / \tau_{e i} m_{i}$. Combining the first two terms on the RHS, we have $\nabla \times \nabla_{\|}(\Psi-\phi)$, where $\nabla_{\|}(\Psi-\phi)$ is the net parallel gradient force on electrons, thereby it is the net parallel gradient force that drives the field line bending.

The electric field in Eq. (2) can be rewritten as ${ }^{42}$

$$
\mathbf{E}=\mathbf{B} \times \mathbf{u}_{F}-\nabla \Psi+\eta \mathbf{j},
$$

where $\mathbf{u}_{F} \equiv B^{-1} \mathbf{b} \times \nabla(\phi-\Psi)=v_{\mathrm{ph} \mid} B_{\perp} / B_{0}=\varsigma u_{E}$ is the velocity of field lines when the resistive magnetic diffusion is absent. Its radial component is the velocity of the magnetic surfaces $\left(\psi_{p}\right)$.

From Faraday's law, we rewrite Alfvén's frozen-in law as

$$
\partial_{t} \mathbf{B}_{\perp}=\nabla \times\left(\mathbf{u}_{F} \times \mathbf{B}\right)+D_{m} \nabla^{2} \mathbf{B}_{\perp}-\nabla \eta \times \mathbf{j} .
$$

The difference between the $\mathbf{E} \times \mathbf{B}$ drift velocity and the field line velocity $\Delta \mathbf{u} \equiv \mathbf{u}_{E}-\mathbf{u}_{F}=B^{-1} \mathbf{b} \times \nabla \Psi$ is a function of the parallel emf $\Psi$. The parallel emf is generally related to some kinetic effects. $^{42}$

It follows that magnetic flux $\Phi \equiv \int_{\Sigma} \mathbf{B} \cdot d \boldsymbol{\sigma}=B \Sigma$ is conserved in the zero electron mass limit, ${ }^{99}$ where $\Sigma$ is the crosssection area of a field-aligned fluid element. The integral form of Alfvén's frozen-in law is

$$
\left(\partial_{t}+\mathbf{u}_{F} \cdot \nabla\right) \Phi=0,
$$

showing that the magnetic flux $\Phi$ is a Lagrangian invariant. The $\mathbf{E} \times \mathbf{B}$ convection of a plasma filament is thus performed in the form of interchange of flux tubes on the timescale smaller than the magnetic diffusion time $\tau_{m} \equiv D_{m}^{-1} L_{\perp}^{2}$ $\sim \tau_{e i} L_{\perp}^{2} / L_{p e}^{2} \sim \tau_{e i} \delta^{-2}$.

The last two terms on the RHS of Eq. (5) are small compared to the other terms except in the far SOL where collisionality is very high. From Table I one can see that the plasma parameters vary significantly across the plasma boundary in a tokamak, which typically involves one order 
of magnitude variations in density and two orders in temperature. As a consequence the collisionality $\nu^{*} \equiv L_{\|} / \lambda_{e}$ changes by more than two orders of magnitude, where $\lambda_{e}$ $\equiv v_{t h e} \tau_{e i}$ is the electron mean free path. The SOL are usually high-collisionality $\left(\nu^{*}>10\right)$ region, and the magnetic diffusion time $\tau_{m}$ is comparable to or even shorter (in the far SOL) than $\tau_{\perp}$, so that the SOL generally belongs to the unfrozen or dissipation region. In the SOL, filaments quickly displace away from the frozen-in flux tubes and drift across field lines due to the magnetic diffusion.

Inside the separatrix $\left(\nu^{*}<10\right)$, the magnetic diffusion time $\tau_{m}$ is much longer than $\tau_{\perp}$, the magnetic diffusion length $L_{m}$ is much shorter than $L_{\perp}$, the magnetic diffusivity $D_{m}<1 \mathrm{~m}^{2} / \mathrm{s}$, and the magnetic Reynolds number $R_{m}$ $\equiv u_{E} L_{\perp} / D_{m} \gg 1$ (see Table I), indicating that the magnetic diffusion, i.e., field lines diffuse across the width $L_{\perp}$ of the filamentary structure, is typically a slow process compared to the transverse convective motion, so that on the timescale of $\tau_{\perp}$ the magnetic diffusion effect is negligible. We can thus refer to the edge as the frozen region. The field lines are dragged away from the unperturbed magnetic field by the plasma filaments at a speed of $\mathbf{u}_{F}$, where $\varsigma \equiv u_{F} / u_{E}<1$.

\section{G. Parallel ion sound wave}

When a plasma filament drifts across the field lines, it may pass through a succession of many magnetic flux tubes. ${ }^{54}$ Such drifting motion creates transient, local disturbance of pressure within the encountered flux tubes, initiating transient parallel transport, i.e., by launching ion sound waves and/or Alfvén waves, away from the position of disturbance. On the timescale of $\tau_{\|}\left(\tau_{\|} \gg \tau_{\perp}\right)$, the pressure perturbations associated with the filament motion drive parallel acoustic response through the parallel motion equation,

$$
\rho d_{t} \mathbf{u}_{\|}=-\nabla_{\|} p_{\|}+\left(p_{\|}-p_{\perp}\right) \nabla_{\|} \ln B-\rho \nu_{\|} \mathbf{u}_{\|},
$$

where $\nu_{\|}$is the kinematic viscosity of the parallel flow, $p_{\|}$ $=p_{i \|}+p_{e \|}$ and $p_{\perp}=p_{i \perp}+p_{e \perp}$. From Eq. (7), we have the timescale of the parallel acoustic response $\sim p u_{\|} L_{\|} / \widetilde{p} c_{s}^{2} \sim \tau_{\|}$ $\sim \delta^{-1} \tau_{\perp}$, where $u_{\|} \sim c_{s}$. As a consequence, on the timescale of $\tau_{\perp}$ the parallel acoustic response is negligible.

\section{COMPRESSIBILITY IN AN INHOMOGENEOUS MAGNETIC FIELD}

Plasma is different from the incompressible neutral fluid in that it is compressible in the directions both parallel and perpendicular to the ambient magnetic field. The parallel compressibility is due to the coupling with ion sound wave; ${ }^{34}$ the perpendicular compressibility is due to the inhomogeneity of the magnetic field, ${ }^{71}$ whereas the magnetic field is incompressible in the case of low- $\beta$, i.e., neglecting the coupling with compressional Alfvén wave. The equation of ion continuity is $d_{t} \ln n=-\nabla \cdot \mathbf{u}_{\perp}-\nabla \cdot \mathbf{u}_{\|} \approx 2 \boldsymbol{\kappa} \cdot \mathbf{u}_{\perp}-\mathbf{B} \cdot \nabla\left(u_{\|} B^{-1}\right)$, where we defined the field line curvature $\boldsymbol{\kappa} \equiv(\mathbf{b} \cdot \nabla) \mathbf{b}=-\mathbf{b}$ $\times(\nabla \times \mathbf{b})$.

\section{A. Poynting's theorem}

In a quasineutral plasma, using $\mu_{0} \mathbf{j}=\nabla \times \mathbf{B}$, we have

$$
\nabla \cdot\left(B^{2} \mathbf{u}_{E}\right)=\mu_{0} \mathbf{j} \cdot \nabla \phi=\mu_{0} \nabla \cdot(\phi \mathbf{j}) .
$$

It can be rewritten as $\nabla \times(\phi \mathbf{B})=\mu_{0} \phi \mathbf{j}-B^{2} \mathbf{u}_{E} \cdot \mu_{0} \phi \mathbf{j}$ and $B^{2} \mathbf{u}_{E}$ are of the same order. We will show that relation (8) is consistent with Poynting's theorem,

$$
\partial_{t} \hat{W}=-\nabla \cdot \mathbf{S}-\mathbf{j} \cdot \mathbf{E},
$$

where $\hat{W} \equiv(1 / 2) \mu_{0}^{-1} B^{2}+(1 / 2) \varepsilon_{0} E^{2} \cong(1 / 2) \mu_{0}^{-1} B^{2}$ is the electromagnetic energy density which is dominated by the magnetic energy density since the electrostatic energy density is negligible in a quasineutral plasma, $\mathbf{S} \equiv \mu_{0}^{-1} \mathbf{E} \times \mathbf{B}=\mu_{0}^{-1} B^{2} \mathbf{u}_{E}$ is the Poynting vector and its divergence is $\nabla \cdot \mathbf{S}$ $=\mu_{0}^{-1} \nabla \cdot\left(B^{2} \mathbf{u}_{E}\right)$. The Joule dissipation term, i.e., the rate at which the electromagnetic fields do work, is $\mathbf{j} \cdot \mathbf{E}=-\mathbf{j} \cdot \nabla \phi$ $-\mathbf{j} \cdot \partial_{t} \mathbf{A}_{\|}$. Substituting $\partial_{t} \hat{W}, \nabla \cdot \mathbf{S}$, and $\mathbf{j} \cdot \mathbf{E}$ into the Poynting's theorem (9), we obtain the energy conservation equation for the electrostatic field $(1 / 2) \varepsilon_{0} \partial_{t} E^{2}+\mu_{0}^{-1} \nabla \cdot\left(B^{2} \mathbf{u}_{E}\right)=\mathbf{j} \cdot \nabla \phi$. With the quasineutral condition, we finally get the same relation as Eq. (8).

With the help of the electric field expression (4), we have the equation of fluctuating magnetic energy,

$$
\begin{aligned}
\partial_{t} \hat{W}_{\perp} & =-\mu_{0}^{-1} \nabla \cdot\left(\mathbf{E} \times \mathbf{B}_{\perp}\right)-j_{\|} E_{\|} \\
& =-\mu_{0}^{-1} \nabla \cdot\left[\left(\mathbf{B} \times \mathbf{u}_{F}+\eta \mathbf{j}\right) \times \mathbf{B}_{\perp}\right]-\eta_{\|} j_{\|}^{2},
\end{aligned}
$$

where $\hat{W}_{\perp} \equiv(1 / 2) \mu_{0}^{-1} B_{\perp}^{2}$. The first term on the RHS is the divergence of the Poynting vector of the KSAW. The last term is the Joule dissipation term. From $\mu_{0} \mathbf{j}_{0}=\nabla \times \mathbf{B}_{0}$, we have

$$
\nabla \cdot\left(B_{0}^{2} \mathbf{u}_{E}\right)=\mu_{0} \mathbf{j}_{0} \cdot \nabla \phi=\nabla \cdot\left(\mu_{0} \phi \mathbf{j}_{0}\right) .
$$

The gradient length on the LHS of Eq. (11) is very long $\sim q R / \in \sim \delta^{-1} R$, but the LHS does not vanish.

\section{B. Compressibility and Lagrangian invariant}

With the low- $\beta$ approximation, ${ }^{76,77}$ i.e., $B_{\perp} / B_{0} \sim \mathrm{s} \delta^{2}$ and $\nabla \cdot\left(B^{2} \mathbf{u}_{E}\right) \ll B^{2} \nabla \cdot \mathbf{u}_{E}$, regarding $B^{2} \mathbf{u}_{E}$ and $\phi \mathbf{j}$ as incompressible, $\nabla \cdot\left(\hat{W} \mathbf{u}_{E}\right)=(1 / 2) \mathbf{j} \cdot \nabla \phi \approx 0$, we can thus write the compression of electric drift as

$$
\begin{aligned}
\nabla \cdot \mathbf{u}_{E} & =B^{-1}(\mathbf{b} \times \nabla \ln B+\nabla \times \mathbf{b}) \cdot \nabla \phi \\
& =2 \Re+\mu_{0} B^{-2} \mathbf{j} \cdot \nabla \phi \\
& \approx 2 \Re,
\end{aligned}
$$

where $\quad \mathfrak{R} \equiv-\mathbf{u}_{E} \cdot \nabla \ln B_{0}=\mathbf{g} \cdot \nabla \phi, \quad \mathbf{g} \equiv B_{0}^{-1} \hat{\mathbf{z}} \times \nabla \ln B_{0}$, $\partial_{\perp} \ln B_{0} \sim R^{-1}$, and $\nabla \times \mathbf{b}=B \mathbf{g}+\mu_{0} B^{-1} \mathbf{j}$. The compressibility is induced by magnetic field curvature and gradient.

In a low- $\beta$ plasma the most unstable perturbations are those inducing minimal magnetic energy variation, i.e., most weakly bending of field lines. On the timescale of $\tau_{\perp}$, although the local magnetic energy density $\hat{W}$ varies with time due to coupling with KSAW, the energy exchange between particles and the equilibrium magnetic field $\mathbf{B}_{0}$ is negligible in a frame moving with the fluid element. This is essentially 
equivalent to say that there is nearly no coupling to compressional Alfvén waves in a low- $\beta$ plasma, hence $W_{0}$ is a Lagrangian invariant,

$$
d_{t} W_{0}=V \nabla \cdot\left(\hat{W}_{0} \mathbf{u}\right)=0,
$$

where we defined the magnetic energy of the equilibrium magnetic field as $W_{0} \equiv V \hat{W}_{0}, V \equiv n^{-1}$ is the volume, and $\hat{W}_{0}$ $\equiv(1 / 2) \mu_{0}^{-1} B_{0}^{2}$ is the magnetic energy density of the equilibrium magnetic field, note $\partial_{t} \hat{W}_{0}=0$.

With Eq. (13) we regard $n B_{0}^{-2}$ as a Lagrangian invariant and $B_{0}^{2} \mathbf{u}$ as an incompressible flow,

$$
\nabla \cdot\left(B_{0}^{2} \mathbf{u}\right)=B_{0}^{2}(\nabla \cdot \mathbf{u}-2 \mathfrak{R}+2 \mathfrak{J})=0,
$$

where $\mathfrak{J} \equiv \mathbf{u}_{\|} \cdot \nabla \ln B_{0}$ denotes the parallel compression, $\partial_{z} \ln B_{0} \sim L_{\|}^{-1}$. For simplicity here we only keep the leading order perpendicular guiding-center velocity $\mathbf{u}_{\perp} \approx \mathbf{u}_{E}$. Using Eq. (14), the continuity equation is written as

$$
d_{t} \ln n=-\nabla \cdot \mathbf{u}=2(\mathfrak{J}-\mathfrak{R}) .
$$

The induction equation (frozen-in equation) can be written in another form, $d_{t}\left(n^{-1} \mathbf{B}\right)=\left(n^{-1} \mathbf{B} \cdot \nabla\right) \mathbf{u}$. Since the RHS usually does not vanish, $n B^{-1}$ is generally not a Lagrangian invariant. Only in 2D models, where magnetic field curvature is neglected, $n B^{-1}$ can be regarded as a Lagrangian invariant and $B \mathbf{u}_{E}$ can be regarded as an incompressible quantity. ${ }^{71,72}$

\section{Compressibility in toroidal geometry}

Now, let us specify this question in the circumstance of toroidal geometry. For simplicity we consider a axisymmetric tokamak with the magnetic field, given by $\mathbf{B}_{0}=B_{c} R_{0} \nabla \varphi$ $+\nabla \varphi \times \nabla \psi_{p}=\left(\mathbf{e}_{\varphi}+\vartheta \mathbf{e}_{\theta}\right) B_{c} R_{0} R^{-1}$, where $B_{c}$ and $R_{0}$ are constants, $B_{c}$ is the center magnetic field value, and $\varphi$ and $\theta$ are the toroidal and poloidal angles of a torus, respectively, $\nabla \varphi$ $=R^{-1} \mathbf{e}_{\varphi}, \psi_{p}$ is the poloidal flux function, $\vartheta=B_{\theta} / B_{\varphi}$ is the ratio between the poloidal and toroidal magnetic fields, and the major radius is given by $R=R_{0}+r \cos \theta$. With such geometry, the time rate of the perpendicular compression is $\gamma_{\perp} \equiv|\Re| \sim u_{E} R^{-1}$, and the parallel gradient is given by $\partial_{z}$ $=k_{c} \partial_{\theta}$, where $k_{c} \equiv(q R)^{-1}$ and $\partial_{\theta} \equiv \partial / \partial \theta$. The time rate of the parallel compression is $\gamma_{\|} \equiv|\mathfrak{J}| \sim \vartheta u_{\|} R^{-1} \sin \theta$. Note $u_{E} / u_{\|}$ $\sim \vartheta \sim \delta$.

On the outboard (low field side $\theta \sim 0$ ) or inboard (high field side $\theta \sim \pi$ ) midplanes, $\sin \theta \sim 0$, the parallel compression $\gamma_{\|}$and the parallel advection $\mathbf{u}_{\|} \cdot \nabla n$ nearly vanish; the perpendicular compression thus dominates over the parallel compression $\gamma_{\perp} \gg \gamma_{\|}$and the continuity equation is reduced to

$$
\hat{d}_{t} \ln n=-2 \Re .
$$

It means that, when a filament is displaced toward regions of weaker background magnetic field, its volume expands at a rate of $\gamma_{\perp}$, and as a result of expansion its density is reduced.

At the top $\theta \sim \pi / 2$ or bottom $\theta \sim-\pi / 2$ of the torus, the parallel and perpendicular compressions are of the same order $\gamma_{\|} \sim \gamma_{\perp}$. The perpendicular compression could be partially canceled by the parallel compression, so that the in- duced density variations in a frame moving with the fluid element could be much smaller than those on the outboard midplane. The spatial dependence of $\gamma_{\|} \sim \gamma_{\perp}$ in a torus is one of the origins of ballooning in toroidal geometry, and as a consequence interchange instabilities mainly arise in the unfavorable curvature region and filaments in the toroidal geometry generally manifest themselves as ballooning structures.

By taking the scalar product with $\mathbf{u}_{\|}$of the parallel momentum Eq. (7), we obtain the equation of parallel kinetic energy evolution,

$$
n d_{t} K_{\|}=-\mathbf{u}_{\|} \cdot \nabla p_{\|}+\left(p_{\|}-p_{\perp}\right) \mathfrak{J}-2 n \nu_{\|} K_{\|},
$$

where $K_{\|} \equiv(1 / 2) m_{i} u_{\|}^{2}$ is the kinetic energy of parallel flows. The second term on the RHS depicts the parallel compression induced by mirror force. The first two terms on the RHS drives ion sound waves along field lines.

On the outboard or inboard midplanes, the parallel compression nearly vanishes, $d_{t} K_{\|} \rightarrow 0$, indicating that the energy transferred into the parallel flows is negligible. Therefore, the dynamics on the outboard or inboard midplanes are nearly $2 \mathrm{D}$. At the top or bottom of the torus, the parallel compression is important, but the mirror force usually counteracts the pressure gradient.

\section{Coupling with kinetic shear Alfvén waves}

The shear Alfvén wave is a transverse wave with electric field and magnetic field perturbations perpendicular to $\mathbf{B}_{0}$. When coupling with KSAW, the field lines are dragged away from the unperturbed magnetic field by the plasma filaments at a speed of $\mathbf{u}_{F}$, where $\varsigma \equiv u_{F} / u_{E}<1$. In a tokamak, the KSAW are launched from the unfavorable curvature region, i.e., the wave source is located on the low field side, where interchange modes are unstable, $\boldsymbol{\kappa} \cdot \nabla\langle p\rangle>0$. The waves propagate along field lines with a phase speed $v_{\mathrm{ph} \|}$. On the high field side interchange modes are stable, $\boldsymbol{\kappa} \cdot \nabla\langle p\rangle\langle 0$. The interchange motion perturbs the field lines in the perpendicular direction, inducing field line bending. A restoring force associated with the resultant force of magnetic tension does work, generating the magnetic energy of field line bending.

In the ideal MHD limit $s=1$, the parallel emf $\Psi$ vanishes, the electric field and magnetic field perturbations in a shear Alfvén wave divide equally the total energy of the electromagnetic wave. ${ }^{100}$ When $\varsigma<1$, we have the magnetic energy of field line bending,

$$
W_{\perp}=\varsigma^{2} K_{\perp},
$$

where $K_{\perp} \equiv(1 / 2) m_{i} u_{\perp}^{2} \approx(1 / 2) m_{i} u_{E}^{2}$ is the perpendicular kinetic energy and $W_{\perp} \equiv V \hat{W}_{\perp}$. For mesoscale structures, $\mathrm{s}$ is of order unity $(\varsigma<1),{ }^{92,93}$ and $W_{\perp}$ and $K_{\perp}$ are of the same order.

Using Eq. (18), we obtain a relation between the parallel current density and the electrostatic vorticity,

$$
j_{\|} \sim \varsigma \mu_{0}^{-1} v_{A}^{-1} B_{0} \Omega,
$$

showing that the parallel current carried by filaments is proportional to the electrostatic vorticity. The amplitudes of magnetic field perturbation and parallel current density mea- 
sured in recent experiments associated with blobs, ${ }^{44,45}$ ELM filaments, ${ }^{46}$ and Alfvén vortex filaments ${ }^{49-52}$ are generally consistent with Eqs. (18) and (19). The importance of the magnetic components was stressed and the relation between the magnetic and electrostatic fluctuation levels was verified in a recent electromagnetic simulation of edge resistive ballooning turbulence. ${ }^{101}$ With the help of Eqs. (13) and (18), we have the energy exchange between particles and the total magnetic field $\mathbf{B}$ in a frame moving with the fluid element,

$$
d_{t} W=d_{t} W_{\perp}=s^{2} d_{t} K_{\perp},
$$

where $W \equiv V \hat{W}=W_{0}+W_{\perp}$ is the total magnetic energy.

\section{GENERATION OF KINETIC ENERGY AND MAGNETIC ENERGY THROUGH THERMAL EXPANSION}

In this section, a general discussion is given of the generation mechanism of drift-Alfvén vortex structures in fusion plasmas.

\section{A. Fundamental thermodynamic relation}

Free energy is stored in the background pressure gradient. The generation of structures requires an effective mechanism to release the free energy and do work so that the thermal energy of particles can be converted into kinetic energy of structure motion and magnetic energy of field line bending. The most important energy transfer channel for a low- $\beta$ plasma is due to the presence of compressibility in an inhomogeneous magnetic field. This process can be interpreted in terms of the fundamental thermodynamic relation

$$
d_{t} U=T d_{t} s-p d_{t} V
$$

and the entropy equation ${ }^{102}$

$$
p d_{t} s=(\boldsymbol{\pi} \cdot \nabla) \cdot \mathbf{u}-\nabla \cdot \mathbf{q}+\mathbf{j} \cdot \mathbf{E},
$$

where $U \equiv C_{v} T=(1 / 2) T_{\|}+T_{\perp}=(1 / 2) m \overline{w^{2}}$ is the plasma internal energy, $T \equiv T_{i}+T_{e}$ is the plasma temperature, $s$ is the specific entropy, $p \equiv n T$ is the plasma pressure, $\boldsymbol{\pi}$ is the offdiagonal tensor of the stress tensor $\mathbf{P} \equiv-p_{\perp} \mathbf{I}-\left(p_{\|}-p_{\perp}\right) \mathbf{b b}$ $+\pi$, and $\mathbf{q}$ is the conductive heat flux. $C_{v}=(\gamma-1)^{-1}$ is the specific heat at constant volume, $C_{p}=\gamma(\gamma-1)^{-1}$ is the specific heat at constant pressure, and $\gamma \equiv C_{p} / C_{v}>1$ is the ratio of specific heats. The particle motion velocity is composed of collective motion velocity and thermal velocity, $\mathbf{v}=\mathbf{u}+\mathbf{w}$. The thermal velocity in a magnetic field is composed of $w^{2}$ $=w_{\|}^{2}+w_{\perp}^{2}$. We defined the parallel temperature $T_{\|} \equiv m \overline{w_{\|}^{2}}$, the perpendicular temperature $T_{\perp} \equiv(1 / 2) m w_{\perp}^{2}$, the parallel pressure $p_{\Perp} \equiv n T_{\|}$, and the perpendicular pressure $p_{\perp} \equiv n T_{\perp}$, where $\overline{w^{2}}$ means average over velocity space.

The first term on the RHS of Eq. (22) describes the viscous dissipation which is dominated by ion viscosity, the second term describes the dissipation due to heat conduction, and the last term describes the Joule dissipation. Note here that the dissipation effect associated with the magnetic diffusion has already been included in Eq. (22).

\section{B. Conservation of specific entropy}

There are several dissipation processes, such as viscosity, heat conduction, and Joule dissipation, which can lead to entropy production, and there are also several different dissipation mechanisms taking place in different situations. In the nearly collisionless plasma core region the entropy production is dominated by nonlinear wave-particle interactions (linear and nonlinear Landau damping) and wave-wave interactions. ${ }^{9}$ In the plasma boundary it could be dominated by collisional dissipation (see Table I), or in the far SOL dominated by sheath dissipation. ${ }^{19-23}$

Whatever the detailed dissipation processes or mechanisms are, we can use a dissipation timescale $\tau_{d}$ to describe the entropy production,

$$
T d_{t} s=\tau_{d}^{-1}\left(K_{\perp}+W_{\perp}\right),
$$

indicating that the effect of dissipation is to consume the kinetic energy of convective motion and the magnetic energy of field line bending and convert them back into the internal energy. This process is irreversible since it is accompanied by entropy production. In many situations of magnetized plasma, the dissipation timescale is much longer than the timescale for perpendicular dynamics, $\tau_{d} \gg \tau_{\perp}$. For instance in a tokamak, except in the far SOL the magnetic diffusion time $\tau_{m}$ is much longer than $\tau_{\perp}$ (see Table I).

Therefore, on the timescale of $\tau_{\perp}$, the dissipation effects are negligible, and the specific entropy is a Lagrangian invariant,

$$
d_{t} s=0 .
$$

Substituting Eq. (24) into Eq. (21), we have

$$
d_{t} U=-p d_{t} V .
$$

It means that the internal energy stored in the background pressure gradient can be tapped off through reversible pressure-volume work. The presence of compressibility in an inhomogeneous magnetic field renders the expansion or compression available. Using Eq. (15), we can rewrite Eq. (25) as

$$
d_{t} U=2(\mathfrak{J}-\mathfrak{R}) T .
$$

On the outboard midplane the parallel compression $\mathfrak{J}$ vanishes, Eq. (26) is thus reduced to

$$
\hat{d}_{t} U=-2 \Re T,
$$

indicating that when a filament is displaced toward regions of weaker background magnetic field and/or in the direction of curvature radius, its internal energy decreases. The reduced internal energy will be converted into kinetic energy and magnetic energy as a result of energy conservation.

\section{Conservation of total energy}

The total energy $H$ (Hamiltonian) is composed of magnetic energy $W$, thermal energy $U$, kinetic energy $K$ $\equiv(1 / 2) m_{i} u^{2}=K_{\|}+K_{\perp}$, and electrostatic energy $W_{E}$ $\equiv(1 / 2) \varepsilon_{0} E^{2} V$. In a low- $\beta$ plasma generally speaking $W$ $\gg U \gg K \gg W_{E}$. In a quasineutral plasma, since $\rho B_{0}^{-2} \gg \varepsilon_{0}$, the kinetic energy of $\mathbf{E} \times \mathbf{B}$ motion is much larger than the elec- 
trostatic energy $K_{\perp} \gg W_{E}$, hence we will hereafter neglect the electrostatic energy. On the timescale of $\tau_{\perp}$, if $\tau_{d} \gg \tau_{\perp}$, all dissipation effects can be neglected.

Now, let us focus on the outboard midplane, where the parallel compression nearly vanishes $d_{t} K_{\|} \rightarrow 0$, thus the conservation of total energy requires

$$
\hat{d}_{t} H=0 \text {, }
$$

where $H \equiv W+U+K_{\perp}$. Combining Eqs. (27) and (28), we have

$$
\hat{d}_{t}\left(K_{\perp}+W_{\perp}\right)=2 \Re T .
$$

This formula depicts the generation of perpendicular kinetic energy and magnetic energy through thermal expansion. From Eq. (29), we get the filament acceleration in the direction of curvature radius $g_{\text {int }} \sim c_{s}^{2} / R$, the interchange acceleration time $\tau_{\text {int }} \sim c_{s}^{-1}\left(R L_{\perp}\right)^{1 / 2}$, and the interchange velocity $u_{\text {int }} \sim c_{s}\left(L_{\perp} / R\right)^{1 / 2} \sim 10 \% c_{s}$. The interchange velocity is the upper limit of filament transverse motion velocity ${ }^{24,25}$ (see Table I).

We can use a simple model to include the dissipation effects,

$$
\hat{d}_{t}\left(K_{\perp}+W_{\perp}\right)=2 \Re T-\tau_{d}^{-1}\left(K_{\perp}+W_{\perp}\right) .
$$

On the timescale of $\tau_{\|}$the parallel kinetic energy $K_{\|}$will participate in the energy partition. In that case part of the filament's energy is transferred into the kinetic energy of parallel flow through the parallel compression, depicted by Eq. (17). With the help of Eq. (16), Eq. (27) can be rewritten in the following forms:

$$
\begin{aligned}
& \hat{d}_{t} \ln p=-2 \gamma \Re, \\
& \hat{d}_{t} \ln T=2(1-\gamma) \Re .
\end{aligned}
$$

With the help of Eq. (13), Eq. (25) can be rewritten in the conservation forms

$$
d_{t}\left(p n^{-\gamma}\right)=d_{t}\left(T n^{1-\gamma}\right)=d_{t}\left(p B_{0}^{-2 \gamma}\right)=d_{t}\left(T B_{0}^{2(1-\gamma)}\right)=0 .
$$

We recognize that $d_{t}\left(p n^{-\gamma}\right)=0$ is just the adiabatic equation.

\section{FILAMENT ACCELERATION AND VORTICITY GENERATION}

In the last section, we explained how the free energy stored in background pressure gradient is released and converted into the kinetic energy of convective motion and the magnetic energy of field line bending associated with the drift-Alfvén vortices. In this section, we will review this process in a dynamic perspective. The acceleration of plasma filament is induced by a force unbalance in the direction of curvature radius resulting from the plasma inertial response on the timescale of $\tau_{\perp}$, reacted to satisfy quasineutrality. A restoring force associated with the resultant force of magnetic tension does work, generating the magnetic energy of field line bending.

\section{A. Vorticity equation}

The plasma current $\mathbf{j}=\mathbf{j}_{f}+\mathbf{j}_{p}+\mathbf{j}_{b}$ is composed of the free current $\mathbf{j}_{f}$, the polarization current $\mathbf{j}_{p}$, and the magnetization (bound) current $\mathbf{j}_{b}$, where the free current $\mathbf{j}_{f}=\mathbf{j}_{\|}+\mathbf{j}_{G}$ can be further decomposed into the parallel current $\mathbf{j}_{\|}$and the magnetic-drift current $\mathbf{j}_{G}$. The polarization current $\mathbf{j}_{p} \equiv e n \mathbf{u}_{p}$ is dominated by the contribution from the ion polarization drift. The magnetization current is defined as $\mathbf{j}_{b} \equiv \nabla \times \mathbf{M}$ $=\mathbf{j}_{d}-\mathbf{j}_{B},{ }^{103}$ where $\mathbf{j}_{d} \equiv B^{-1} \mathbf{b} \times \nabla p_{\perp}$ is the diamagnetic current and $\mathbf{j}_{B} \equiv B^{-1} p_{\perp}(\mathbf{b} \times \nabla \ln B+\nabla \times \mathbf{b})$ is a component of the magnetic-drift current. The magnetic-drift current is induced by the oppositely directed guiding center drifts of ions and electrons in an inhomogeneous magnetic field $\mathbf{j}_{G} \equiv B^{-1}\left(p_{\perp} \mathbf{b}\right.$ $\left.\times \nabla \ln B+p_{\|} \nabla \times \mathbf{b}\right)=\mathbf{j}_{B}+\mathbf{j}_{a}$, where $\mathbf{j}_{a} \equiv B^{-1}\left(p_{\|}-p_{\perp}\right) \nabla \times \mathbf{b}$ is induced by anisotropy. The total perpendicular current density can be approximately written in the form $\mathbf{j}_{\perp} \cong \mathbf{j}_{b}+\mathbf{j}_{G}$ $+\mathbf{j}_{p}=\mathbf{j}_{d}+\mathbf{j}_{a}+\mathbf{j}_{p}$.

Noting $\nabla \cdot \mathbf{j}_{b}=0$, we have the quasineutral condition

$$
\nabla \cdot \mathbf{j}_{\perp}+\nabla \cdot \mathbf{j}_{\|}=\nabla \cdot \mathbf{j}_{p}+\nabla \cdot \mathbf{j}_{G}+B \partial_{\|}\left(j_{\|} B^{-1}\right)=0,
$$

where the compression of magnetic-drift current is given by

$$
\begin{aligned}
\nabla \cdot \mathbf{j}_{G}= & \mathbf{g} \cdot \nabla\left(p_{\perp}+p_{\|}\right) \\
& +\mu_{0} B^{-2} \mathbf{j} \cdot\left[\nabla p_{\|}+\left(p_{\perp}-p_{\|}\right) \nabla \ln B\right] \\
\approx & \mathbf{g} \cdot \nabla\left(p_{\perp}+p_{\|}\right) .
\end{aligned}
$$

With the Boussinesq approximation ${ }^{105}$ the compression of polarization current can be written as

$$
\nabla \cdot \mathbf{j}_{p} \approx \rho d_{t}\left(\Omega B^{-1}\right) .
$$

The compression of diamagnetic current is given by

$$
\nabla \cdot \mathbf{j}_{d}=\nabla \cdot \mathbf{j}_{B}=2 \mathbf{g} \cdot \nabla p_{\perp}+\mu_{0} B^{-2} \mathbf{j} \cdot \nabla p_{\perp} \approx 2 \mathbf{g} \cdot \nabla p_{\perp} .
$$

Inserting Eqs. (35) and (36) into the quasineutral condition (34), we arrive at the vorticity equation ${ }^{24,25}$

$$
\rho d_{t}\left(\Omega B^{-1}\right)=B \partial_{\|}\left(j_{\|} B^{-1}\right)+\mathbf{g} \cdot \nabla\left(p_{\perp}+p_{\|}\right) .
$$

Another approach to get the vorticity equation is by taking curl of the motion equation,

$$
\begin{aligned}
\rho d_{t} \mathbf{u}= & \mathbf{j} \times \mathbf{B}-\nabla_{\perp} p_{\perp}-\nabla_{\|} p_{\|}-\left(p_{\|}-p_{\perp}\right)\left(\boldsymbol{\kappa}-\nabla_{\|} \ln B\right) \\
& +\nabla \cdot \boldsymbol{\pi},
\end{aligned}
$$

and forming the scalar product with $\mathbf{b} .{ }^{106}$ The field line curvature is $\boldsymbol{\kappa}=\nabla_{\perp} \ln B+\mu_{0} B^{-2} \mathbf{j} \times \mathbf{B}$ and $\mathbf{b} \times \boldsymbol{\kappa}=(\nabla \times \mathbf{b})_{\perp}$ $=B \mathbf{g}+\mu_{0} B^{-1} \mathbf{j}_{\perp}$. With the Boussinesq approximation, ${ }^{105}$ we obtain the same vorticity equation as Eq. (38). ${ }^{24,25}$

The last term in Eq. (38) is induced by the perpendicular compressibility. This term is the same term as that on the RHS of Eq. (29). This term releases the free energy stored in background pressure gradient and converts them into the kinetic energy of convective motion resulting in vorticity generation, and into the magnetic energy of field line bending through the parallel current. The parallel current is contained in the first term on the RHS of Eq. (38). One can see that the physics described by Eq. (29) is essentially the same as those in the vorticity Eq. (38). 


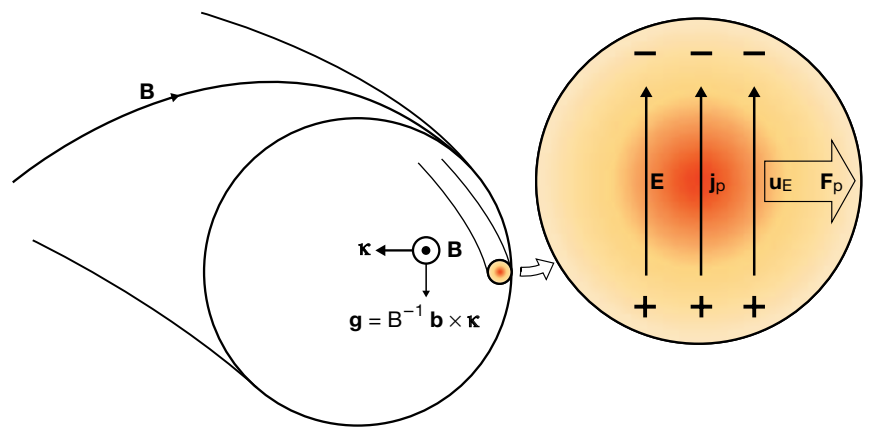

FIG. 6. (Color) Illustration of the interchange mechanism leading to radial motion of a filamentary structure in tokamak geometry. The vertical polarization and vorticity due to magnetic guiding center drifts results in a radial electric drift at the center of the filamentary structure and a net force due to the vertical polarization current drives the filament to accelerate in the direction of curvature radius, which is followed by the formation of dipolar vorticity and field line bending.

If the plasma is incompressible, the RHS of Eq. (38) will vanish, $d_{t}\left(\Omega B^{-1}\right)=0$. With the help of the magnetic flux invariant, $d_{t} \Phi=0$, we have $I \equiv \int_{\Sigma} \boldsymbol{\Omega} \cdot d \boldsymbol{\sigma}=\Omega \Sigma=$ const. It is just the integral form of Kelvin's frozen-in law for incompressible fluid. ${ }^{68}$ Its differential form is $\partial_{t} \boldsymbol{\Omega}=\nabla \times(\mathbf{u} \times \boldsymbol{\Omega})$, implying that vortices are frozen in fluid elements and moving with them. In a compressible fluid this law is violated.

The interchange mechanism leading to radial motion of a filamentary structure is illustrated in Fig. 6. In tokamak geometry $\mathbf{g}$ is in the vertical direction, so that the charge polarization induced by the magnetic guiding center drifts is in the vertical direction, leading to a radial electric drift at the center of the filamentary structure, ${ }^{19-25}$ and a net force due to the vertical polarization current drives the filament to accelerate in the direction of curvature radius, which is followed by the formation of dipolar vorticity ${ }^{24,25}$ and field line bending. 33

\section{B. Equivalent circuit}

From the quasineutral condition (34), one can see that the curvature and grad-B drifts function as a local current source. The currents generated by this current source flow along and across the field lines compete through their effective impedance and establish current loops, ${ }^{27,107}$ as illustrated in the following equivalent circuit diagram.

Plasma inertia plays the role of a capacitor. The current flowing through the capacitor is the polarization current. This current is responsible for filament acceleration and accumulation of perpendicular kinetic energy. All dissipation processes, such as the Joule dissipation, viscous damping, nonlinear wave-particle Landau interaction, or sheath dissipation, can be represented by an equivalent resistor, as shown in Fig. 7. The dissipation processes are irreversible since they are accompanied by entropy production. Parallel current provides a channel to couple the electrostatic vortex dynamics with the KSAW. The coupling process is similar to that occurring in an inductor, as illustrated in Fig. 7. Magnetic energy is accumulated in the inductor during this process. The inductance of a filament is of the order of $\mu_{0} L_{\|}$.

In the electrostatic limit $(\varsigma \ll 1)$, there is nearly no field

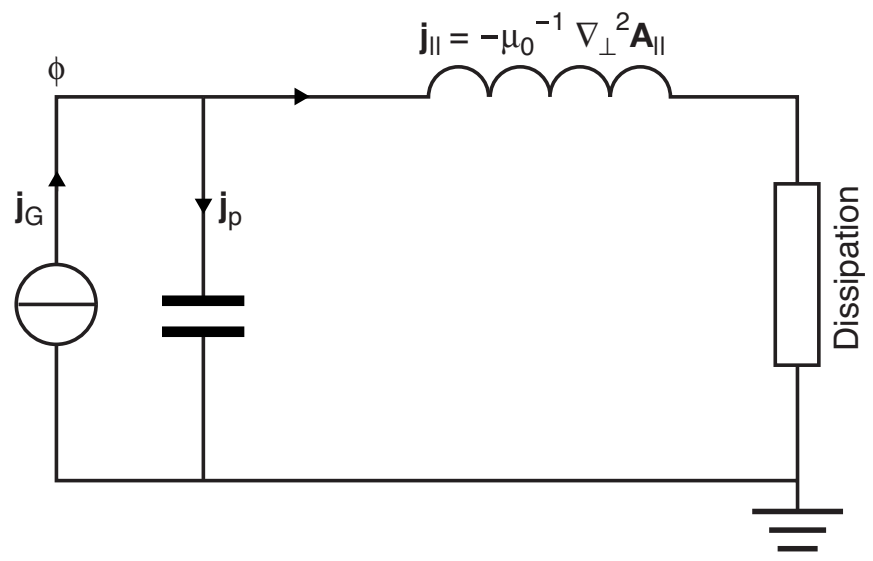

FIG. 7. The equivalent circuit diagram for an Alfvén vortex filament.

line bending. This special case corresponds to a very small equivalent inductance, in a tokamak it implies $L_{\|} \ll \pi q R$ and $L_{\perp} \ll \rho_{\theta i}$, showing that the electrostatic limit is more applicable to smallscale structures. For mesoscale structures, $s$ $\sim 1, L_{\|} \sim \pi q R$, and $L_{\perp} \gg \rho_{s}$, therefore the mesoscale structures are essentially electromagnetic, and compared with small scale structures they are more close to the MHD limit.

In the ideal MHD limit ( $s=1)$, the parallel emf $\Psi$ vanishes and the induction part of parallel electric field cancels the electrostatic part, leading to vanishing parallel electric field $E_{\|}=-\partial_{\|} \phi-\partial_{t} A_{\|} \rightarrow 0$. The parallel streaming of adiabatic electrons is strongly impeded by the magnetic induction so that the neutralization along the field lines is incomplete. For detailed discussion about the magnetic induction effects, please see Sec. III E.

\section{Plasma equilibrium}

On the timescale of plasma equilibrium $\tau_{\mathrm{eq}} \gg \tau_{\perp}$, the plasma inertial term $\left(\nabla \cdot \mathbf{j}_{p 0} \propto \tau_{\text {eq }}^{-1}\right)$ is negligible, then the quasineutral condition is reduced to $\nabla \cdot \mathbf{j}_{G 0}+\nabla \cdot \mathbf{j}_{\| 0}=0$, i.e., $\mathbf{g} \cdot \nabla\left(p_{\perp 0}+p_{\| 0}\right)=-B_{0} \partial_{\|}\left(j_{\| 0} B_{0}^{-1}\right)$. In tokamak plasmas it means that an electric charge separation in the vertical direction induced by magnetic guiding center drifts (see Fig. 6) is neutralized by a parallel return current, i.e., the so called Pfirsch-Schlüter current. The rotation transformation of field lines in tokamak geometry guarantees the current loop is closed and the neutralization is nearly accomplished (except a small neoclassical parallel electric field $\left.E_{\| 0}=\eta_{\|} j_{\| 0}\right)$. The equilibrium equation is given by $\nabla\left\lfloor\hat{W}_{0}(1+\beta)\right\rfloor$ $=\mu_{0}^{-1}\left(\mathbf{B}_{0} \cdot \nabla\right) \mathbf{B}_{0}$, indicating that the gradient of magnetic pressure plus thermal pressure is balanced by the resultant force (in the curvature direction) of the magnetic tension (along field line) due to the field line curvature $\boldsymbol{\kappa}_{0}=\nabla_{\perp} \ln B_{0}$ $+\mu_{0} B_{0}^{-2} \nabla_{\perp} p_{0} \sim(1+\beta) \nabla_{\perp} \ln B_{0}$. This force acts as a restoring force in the filamentary dynamics.

\section{Filament acceleration}

Plasma has a natural tendency to expand in the direction of curvature radius in an inhomogeneous magnetic field. The thermal expansion force is usually referred to as an effective gravity, ${ }^{33}$ 


$$
\begin{aligned}
\mathbf{F}_{G} & \equiv-\left(p_{\perp}+p_{\|}\right) \nabla_{\perp} \ln B-p_{\|} \mu_{0} B^{-2} \mathbf{j} \times \mathbf{B} \\
& \approx-\left(p_{\perp}+p_{\|}\right) \boldsymbol{\kappa} .
\end{aligned}
$$

On the equilibrium timescale this thermal expansion force is balanced by a magnetic force, i.e., the resultant force of the magnetic tension $\mathbf{F}_{B 0}=\mathbf{j}_{G 0} \times \mathbf{B}_{0}=-\mathbf{F}_{G 0}$.

On the timescale of interchange motion $\tau_{\perp}$ the plasma inertia term $\nabla \cdot \mathbf{j}_{p}$ becomes important. In tokamak geometry the magnetic guiding center drifts induce a local charge polarization in the vertical direction (see Fig. 6). There are several mechanisms impeding the parallel motion of free electrons, such as magnetic induction, magnetic trapping, electron-ion collisions, electron inertia, and wave-electron Landau interaction, so that the parallel motion of electrons cannot completely neutralize the charge polarization on the short timescale $\tau_{\perp}$. Thus, a polarization current $\mathbf{j}_{p}$ in the vertical direction (see Fig. 6) will react to satisfy the local quasineutrality. The vertical charge polarization and the resultant vertical electric field leads to a radial $\mathbf{E} \times \mathbf{B}$ drift at the center of the filamentary structure (see Fig. 6), which drives plasma filament to move in the direction of curvature radius. This interchange mechanism for filament motion plays a central role in the $2 \mathrm{D}$ theoretical models of blob ${ }^{19-23}$ and was highlighted in our recent work. ${ }^{24,25,86-89}$

From the dynamic point of view, the filament acceleration is induced by a force unbalance in the direction of curvature radius. The magnetic guiding center drift current $\mathbf{j}_{G}$ is locally partially canceled by the vertical polarization current $\mathbf{j}_{p}$, so that the local magnetic force is reduced $\mathbf{F}_{B}=\left(\mathbf{j}_{G}+\mathbf{j}_{p}\right)$ $\times \mathbf{B}$, which can no longer balance the thermal expansion force $\mathbf{F}_{G}$, and then the net force

$$
\mathbf{F}_{p}=\mathbf{F}_{G}+\mathbf{F}_{B}=\mathbf{j}_{p} \times \mathbf{B}
$$

will push plasma to accelerate in the direction of curvature radius. During this process the thermal expansion force does work,

$$
\mathbf{u}_{E} \cdot \mathbf{F}_{G}=\mathbf{j}_{G} \cdot \nabla_{\perp} \phi=n \hat{d}_{t}\left(K_{\perp}+W_{\perp}\right)=2 \Re p,
$$

where Eq. (29) has been used. As a consequence, the thermal energy stored in the background pressure gradient is tapped off. Note that only part of the work is converted into the kinetic energy of convective motion, leading to the filament acceleration and electrostatic vorticity generation. This part of work is done by the net force.

$$
\mathbf{u}_{E} \cdot \mathbf{F}_{p}=n \hat{d}_{t} K_{\perp} .
$$

The other part of the work is converted into the magnetic energy of field line bending, and resulting in the generation of magnetic vorticity $\boldsymbol{\Omega}_{A} \equiv \nabla \times \mathbf{B}_{\perp}$. This part of work is done by the restoring force, i.e., the resultant force of the magnetic tension

$$
\mathbf{u}_{E} \cdot \mathbf{F}_{B}=-n \hat{d}_{t} W_{\perp} .
$$

\section{CROSS-FIELD TRANSPORT DUE TO THE VIOLATION OF THE THIRD ADIABATIC INVARIANCE}

In this section we discuss the fundamental kinetic mechanism responsible for the cross-field transport associated with the filamentary dynamics. If the particle system of a magnetized plasma is completely adiabatic, that is to say, there is no energy exchange between particles and electromagnetic field and no collision or other dissipation process, particle orbits are exactly determined by several adiabatic invariants. In tokamak geometry there are three such adiabatic invariants: $\boldsymbol{\mu} \equiv-(1 / 2) \mathbf{b} B^{-1} m v_{\perp}^{2}$ is the magnetic moment (the action of the gyromotion), $J \equiv \oint m v_{\|} d l_{\|}$is the longitudinal invariant (the action of parallel bounce), and $\psi_{p}$ is the poloidal magnetic flux (the action of procession) which defines magnetic flux surfaces. If the particle system is completely adiabatic, the three actions are conserved and no particle can escape from the geometry space confined by the magnetic field. Each adiabatic invariant corresponds to a type of periodic motion. The period of the gyromotion is $\tau_{c}$, the period of the bounce motion is $\tau_{b}$, the period of toroidal procession is $\tau_{p}$, and usually $\tau_{p} \gg \tau_{b} \gg \tau_{c}$.

In a quasineutral plasma the electrostatic energy $W_{E}$ is so small that the energy exchange between particles and electrostatic field is negligible. If the timescale for energy exchange between particles and magnetic field is shorter than or comparable with the period of an adiabatic motion, the corresponding adiabatic invariant will be violated.

From Eqs. (20), (29), and (44), we have known that the timescale for the energy exchange between particles and magnetic field, i.e., the timescale for field line bending, is the timescale for interchange motion $\tau_{\perp}$. This timescale is typically short compared to the period of toroidal procession $\tau_{p}$, implying that the magnetic flux invariant $\psi_{p}$ is not conserved during this process, and, as a consequence, particles can escape across the nested magnetic flux surfaces.

Note that filaments are localized structures with finite toroidal mode number. When a filament is generated, it will induce local field line bending, which is accompanied by $\mathbf{B}$ toroidal symmetry breaking on the timescale of $\tau_{\perp}$. This is one of the fundamental kinetic mechanisms for cross-field turbulent transport in a toroidal magnetic confinement system.

\section{LAGRANGIAN INVARIANTS}

In this section we summarize the involved Lagrangian invariants. The filament motion is controlled by six Lagrangian invariants. The first Lagrangian invariant is the magnetic flux, introduced in Eq. (6). The second is the magnetic energy of the equilibrium magnetic field $\mathbf{B}_{0}$, introduced in Eq. (13). The third is the specific entropy, introduced in Eq. (24). The fourth is the total energy (Hamiltonian), introduced in Eq. (28). The other two are magnetic momentum and toroidal angular momentum.

\section{A. Conservation of magnetic momentum}

The timescale for interchange motion is much longer than the ion gyroperiod $\tau_{\perp} \gg \tau_{c i}$, so that the adiabatic condi- 
tion for gyromotion is satisfied and the magnetic moment $\mu$ is an adiabatic invariant. Also, the ion-ion collision time $\tau_{i i}$, the ion temperature isotropization time $\tau_{T i}$, and the electronion energy exchange time $\tau_{e x}$ are much longer than $\tau_{\perp}$ (see Table I), thus the magnetic momentum of ion is conserved. But for electrons, in most of the boundary region, the electron-ion collision time $\tau_{e i}$ and the electron temperature isotropization time $\tau_{T e}$ are shorter than $\tau_{\perp}$. Therefore, in the plasma boundary region, the average magnetic momentum $\bar{\mu}=T_{\perp} B^{-1}$ is generally a Lagrangian invariant for ions but not for electrons, where the average is over velocity space.

$$
d_{t} \bar{\mu}=0 \text {. }
$$

With the help of Eq. (33), we find when the temperature is isotropic, $T_{\|}=T_{\perp}=T$, the ratio of specific heats is $\gamma=5 / 3$. The corresponding degrees of freedom is $N=2(\gamma-1)^{-1}=3$. Using Eq. (25), (33), and (45), we obtain the Chew, Goldberger, and Low (CGL) double adiabatic equations, ${ }^{108}$

$$
d_{t}\left(p_{\perp} B^{-1} n^{-1}\right)=d_{t}\left(p_{\|} B^{2} n^{-3}\right)=0 .
$$

On the outboard midplane, with the help of Eqs. (16), (27), (31), and (32), Eq. (46) can be rewritten in the following forms:

$$
\begin{aligned}
& \hat{d}_{t} U=-\left(T_{\perp}+T_{\|}\right) \Re, \\
& \hat{d}_{t} \ln T_{\perp}=-\Re, \\
& \frac{1}{2} \hat{d}_{t} \ln T_{\|}=-\Re, \\
& \hat{d}_{t} \ln p_{\perp}=-3 \Re, \\
& \frac{1}{2} \hat{d}_{t} \ln p_{\|}=-2 \Re .
\end{aligned}
$$

\section{B. Conservation of toroidal angular momentum}

In toroidal configuration the toroidal angular momentum density $L_{\varphi}$ is a conserved quantity. ${ }^{109}$ Similar to the equation of continuity (15), we have the conservation equation of $L_{\varphi}{ }^{76,77}$

$$
d_{t} \ln L_{\varphi}=-\nabla \cdot \mathbf{u}=2(\mathfrak{J}-\mathfrak{R}) .
$$

Combining the continuity Eqs. (15) and (52), we find that the toroidal angular momentum $\ell_{\varphi} \equiv V L_{\varphi}=\hat{R} u_{\varphi}$ is a Lagrangian invariant,

$$
d_{t} \ell_{\varphi}=d_{t}\left(L_{\varphi} B_{0}^{-2}\right)=d_{t}\left(\hat{R} u_{\varphi}\right)=0
$$

where $\hat{R} \equiv\left\langle R^{2}\right\rangle /\langle R\rangle$ is an effective major radius and it is an increasing function of $r$ (minor radius), and $u_{\varphi}$ is the toroidal rotation velocity. For a simple torus with concentric circular flux surfaces, $\hat{R}^{2} \sim R_{0}^{2}\left(1+(3 / 2) \in^{2}\right)$. ${ }^{76}$ The moment of inertia density is related to the effective major radius through $I$ $\equiv \rho \hat{R}^{2}$, which suggests that the core is less inert, i.e., lighter, than the edge, so if the toroidal angular momentum $\ell_{\varphi}$ is homogenized by turbulence, the rotation near the core will be faster than at the edge. Because of this $r$ dependence of the moment of inertia density in toroidal geometry, the linear toroidal momentum density $P_{\varphi} \equiv \rho u_{\varphi}=L_{\varphi} / \hat{R}$ is not conserved. With the definition of the angular velocity $\omega_{\varphi}$ $\equiv \hat{R}^{-1} u_{\varphi}$, the toroidal angular momentum density can be expressed as $L_{\varphi}=I \omega_{\varphi}$. From Eq. (53), using the relation between the toroidal angular momentum density and the linear toroidal momentum density, we have $d_{t}\left(P_{\varphi} \hat{R} B_{0}^{-2}\right)=0$.

Assuming filaments carry toroidal momentum, Eq. (53) implies that, when a filament is displaced outwards, $\hat{R}$ increases, and thus the toroidal rotation velocity $u_{\varphi}$ will be reduced. On the contrary, when a filament is displaced inwards, $u_{\varphi}$ will increase. This is an anomalous pinch effect, which has recently been proposed to explain the phenomena of the so-called intrinsic/spontaneous rotation in the plasma core. $^{76,77}$ It was observed in recent experiments ${ }^{110}$ that the rotation profiles were peaked in the plasma core without external momentum injection. In general, an anomalous "pinch" effect is required to explain this observation.

On the outboard midplane, similar to Eq. (16), Eq. (52) is reduced to

$$
\hat{d}_{t} \ln L_{\varphi}=-2 \Re .
$$

One can see that the behaviors of toroidal angular momentum density are very similar to the particle density. ${ }^{76,77}$

\section{CURVATURE-DRIVEN CONVECTIVE TRANSPORT AND ANOMALOUS PINCH EFFECTS}

A strongly intermittent nature of cross-field transport of particles and heat in the boundary region of fusion plasmas has been recognized for more than one decade. ${ }^{2,3}$ There are strong indications that this is caused by field-aligned filamentary structures in the form of plasma blobs propagating radially far into the SOL. ${ }^{33}$ In this paper we suggested that these structures can be associated with the drift-Alfvén vortices. The intermittent convective transports of particles, heat, and momentum across magnetic field can be interpreted in terms of the ballistic motion of these solitary filamentary structures. To quantitatively calculate the resultant transport fluxes, we need nonlinear electromagnetic turbulence simulations, ${ }^{35-37,101}$ but for a qualitative estimation we can use the quasilinear method. ${ }^{76}$ The mode-independent part of the curvature-driven turbulent convective pinch of particles, heat, and momentum ${ }^{111}$ are briefly reviewed in this section. The phenomena of profile consistency, or resilience, are generally thought to be associated with these anomalous pinch effects. ${ }^{112}$ We divide the discussion below into three subsections according to different transport categories.

\section{A. Particle transport}

To simplify the further analytic process we use the tokamak configuration described in Sec. IV C. The radial gradient of the background magnetic field $B_{0}=B_{c}(1+\in \cos \theta)^{-1}$ is $\partial_{r} B_{0}^{\xi}=-\xi B_{0}^{\xi} R^{-1} \cos \theta$. The symbol for perpendicular compressibility $\mathfrak{R} \approx(1 / 2) \nabla \cdot \mathbf{u}_{E}$ can be expressed as $\mathfrak{R}=u_{R} R^{-1}$ $=u_{r} R^{-1} \cos \theta$, where $u_{R}$ is a component of $\mathbf{u}_{E}$ in the direction of curvature radius and $u_{r}$ is the radial component. Assuming the perpendicular kinetic energy associated with the filamentary structures bears ballooning distribution like $K_{\perp}=f(\theta)$ 
$\times\left\langle K_{\perp}\right\rangle$, where $f(\theta) \equiv \alpha^{-1} \pi^{-1 / 2} \exp \left(-\theta^{2} / \alpha^{2}\right)$ is the poloidal distribution function and $\alpha$ is a factor characterizing ballooning degree. We note that the poloidal distribution function is a weighting function, then we have $\left\langle K_{\perp} R^{-1} \cos \theta\right\rangle /\left\langle K_{\perp}\right\rangle$ $\approx R_{\text {out }}^{-1}$, where $R_{\text {out }} \equiv R_{0}+r$ denotes the major radius on the outboard side (low-field side).

Following the quasilinear method, ${ }^{76}$ separating $n=\langle n\rangle$ $+\tilde{n}$, substituting it into Eq. (13), we have $\partial_{t} \tilde{n}$ $+B_{0}^{2} \widetilde{u}_{r} \partial_{r}\left(\langle n\rangle B_{0}^{-2}\right)=0$, indicating that $n B_{0}^{-2}$ is conserved in the convective process. Note the timescale for density variation is $\tau_{\perp}$. Then, the density perturbation can be expressed as

$$
\widetilde{n}=-\tau_{\perp} \widetilde{u}_{r} \partial_{r}\langle n\rangle-2 \tau_{\perp}\langle n\rangle \widetilde{u}_{r} R^{-1} \cos \theta .
$$

Substituting the density perturbation (55) into the radial convective particle flux,

$$
\Gamma_{n} \equiv\left\langle\tilde{n} \tilde{u}_{r}\right\rangle=-D \partial_{r}\langle n\rangle+V_{n r}\langle n\rangle,
$$

we get the turbulent diffusivity $D \equiv m_{i}^{-1} \tau_{\perp}\left\langle K_{\perp}\right\rangle$ and the curvature-driven pinch velocity $V_{n r} \equiv-2 R_{\text {out }}^{-1} D$, where we rendered $K_{\perp}=m_{i} \widetilde{u}_{r}^{2}$. This anomalous pinch effect is induced by curvature-driven convection, as a consequence the pinch velocity is proportional to $R_{\text {out }}^{-1}$. Both the turbulent diffusivity and the anomalous pinch velocity are proportional to the fluctuation intensity $\left\langle K_{\perp}\right\rangle$.

Note that there is no offdiagonal term in Eq. (56), such as the temperature gradient term. According to the principle of Onsager symmetries there should be no off diagonal term in the temperature transport equations. It was suggested that, when the FLR effects are taken into account, off diagonal terms will appear in the transport matrix. ${ }^{72}$ Substituting the radial particle flux (56) into the particle transport equation,

$$
\partial_{t}\langle n\rangle+r^{-1} \partial_{r}\left(r \Gamma_{n}\right)=0
$$

we finally arrive at the density evolution equation. With this equation one can calculate density profile and its evolution.

\section{B. Thermal transport}

Following the same procedure as in Sec. IX A, we separate $p=\langle p\rangle+\widetilde{p}$ and $T=\langle T\rangle+\widetilde{T}$. Substituting them into Eq. (33), we have $\partial_{t} \widetilde{p}+B_{0}^{2 \gamma} \widetilde{u}_{r} \partial_{r}\left(\langle p\rangle B_{0}^{-2 \gamma}\right)=0$ and $\partial_{t} \widetilde{T}$ $+B_{0}^{2(\gamma-1)} \tilde{u}_{r} \partial_{r}\left(\langle T\rangle B_{0}^{2(1-\gamma)}\right)=0$, indicating that $p B_{0}^{-2 \gamma}$ and $T B_{0}^{2(1-\gamma)}$ are conserved in the convection process. The pressure perturbation and the temperature perturbation can be written as

$$
\begin{aligned}
& \tilde{p}=-\tau_{\perp} \tilde{u}_{r} \partial_{r}\langle p\rangle-2 \gamma \tau_{\perp}\langle p\rangle \tilde{u}_{r} R^{-1} \cos \theta, \\
& \tilde{T}=-\tau_{\perp} \tilde{u}_{r} \partial_{r}\langle T\rangle-2(\gamma-1) \tau_{\perp}\langle T\rangle \widetilde{u}_{r} R^{-1} \cos \theta .
\end{aligned}
$$

Substituting the temperature perturbation (59) into the radial conductive thermal flux,

$$
\Gamma_{T} \equiv\left\langle\widetilde{T} \widetilde{u}_{r}\right\rangle=-D \partial_{r}\langle T\rangle+V_{T r}\langle T\rangle,
$$

where $D$ is still the turbulent diffusivity and $V_{T r} \equiv-2(\gamma$ $-1) R_{\text {out }}^{-1} D$ is the conductive thermal pinch velocity. Substituting the pressure perturbation (58) into the radial thermal flux (convective plus conductive thermal flux),

$$
\Gamma_{p} \equiv\left\langle\widetilde{p} \widetilde{u}_{r}\right\rangle=-D \partial_{r}\langle p\rangle+V_{p r}\langle p\rangle=\langle T\rangle \Gamma_{n}+\langle n\rangle \Gamma_{T},
$$

where $V_{p r} \equiv-2 \gamma R_{\text {out }}^{-1} D$ is the anomalous thermal pinch velocity.

Equations (31) and (32) can be rewritten as

$$
\begin{aligned}
& \partial_{t} p+\nabla \cdot\left(p \mathbf{u}_{E}\right)=2(1-\gamma) p \Re, \\
& \partial_{t} T+\nabla \cdot\left(T \mathbf{u}_{E}\right)=2(2-\gamma) T \Re .
\end{aligned}
$$

Note Eqs. (31) and (32) are written on the outboard midplane. Inserting Eqs. (58) and (59) into Eqs. (62) and (63) and taking average over a flux surface, we obtain the thermal transport equations,

$$
\begin{aligned}
& \partial_{t}\langle p\rangle+r^{-1} \partial_{r}\left(r \Gamma_{p}\right)=(1-\gamma) V_{n r}\left(\partial_{r}\langle p\rangle-V_{p r} D^{-1}\langle p\rangle\right), \\
& \partial_{t}\langle T\rangle+r^{-1} \partial_{r}\left(r \Gamma_{T}\right)=(2-\gamma) V_{n r}\left(\partial_{r}\langle T\rangle-V_{T r} D^{-1}\langle T\rangle\right) .
\end{aligned}
$$

Using these thermal transport equations, one can calculate the self-consistent profiles of pressure and temperature.

\section{Toroidal momentum transport}

It was pointed out in a recent research ${ }^{76,77}$ that the behaviors of toroidal angular momentum density $L_{\varphi}$ are very similar to the particle density since they are both conserved quantities. Similar to the procedure in Sec. IX A, we obtain the $L_{\varphi}$ flux,

$\Gamma_{L}=-D \partial_{r}\left\langle L_{\varphi}\right\rangle+V_{n r}\left\langle L_{\varphi}\right\rangle$,

and the $L_{\varphi}$ transport equation,

$$
\partial_{t}\left\langle L_{\varphi}\right\rangle+r^{-1} \partial_{r}\left(r \Gamma_{L}\right)=0 .
$$

As noted, ${ }^{76,77}$ it is the toroidal flow velocity $u_{\varphi}=L_{\varphi} \rho^{-1} \hat{R}^{-1}$ rather than the toroidal angular momentum density $L_{\varphi}$ that is measured in experiments. The toroidal velocity profiles can be directly measured by charge exchange recombination spectroscopy diagnostics. ${ }^{110}$ In order to write the evolution of toroidal flow, we need to disentangle the flow and the density.

Separating $u_{\varphi}=\left\langle u_{\varphi}\right\rangle+\tilde{u}_{\varphi}$, substituting it into Eq. (53), we have $\partial_{t} \widetilde{u}_{\varphi}+\hat{R}^{-1} \widetilde{u}_{r} \partial_{r}\left(\hat{R}\left\langle u_{\varphi}\right\rangle\right)=0$. Then, the toroidal velocity perturbation is

$$
\tilde{u}_{\varphi}=-\tau_{\perp} \widetilde{u}_{r} \partial_{r}\left\langle u_{\varphi}\right\rangle-\tau_{\perp} \tilde{u}_{r}\left\langle u_{\varphi}\right\rangle \hat{R}^{-1} \partial_{r} \hat{R} .
$$

Substituting the toroidal velocity perturbation (68) into the radial convective flux

$$
\Gamma_{\varphi} \equiv\left\langle\tilde{u}_{\varphi} \tilde{u}_{r}\right\rangle=-D \partial_{r}\left\langle u_{\varphi}\right\rangle+V_{\varphi r}\left\langle u_{\varphi}\right\rangle,
$$

where $V_{\varphi r} \equiv-m_{i}^{-1} \tau_{\perp}\left\langle K_{\perp} \hat{R}^{-1} \partial_{r} \hat{R}\right\rangle$ is the anomalous pinch velocity of the toroidal velocity. Rewriting Eq. (53) as

$$
\partial_{t} u_{\varphi}+\nabla \cdot\left(u_{\varphi} \mathbf{u}\right)=-u_{\varphi} u_{r} \hat{R}^{-1} \partial_{r} \hat{R}+2 u_{\varphi} \mathfrak{R} .
$$

Averaging Eq. (70) over a flux surface, and with the help of Eq. (68), we get the transport equation for the toroidal velocity, 


$$
\begin{aligned}
\partial_{t}\left\langle u_{\varphi}\right\rangle+r^{-1} \partial_{r}\left(r \Gamma_{\varphi}\right)= & \left(V_{n r}-V_{\varphi r}\right) \partial_{r}\left\langle u_{\varphi}\right\rangle \\
& +m_{i}^{-1} \tau_{\perp}\left\langle K_{\perp} \hat{R}^{-2}\left(\partial_{r} \hat{R}\right)^{2}\right. \\
& \left.-2 K_{\perp} R^{-1} \cos \theta \hat{R}^{-1} \partial_{r} \hat{R}\right\rangle\left\langle u_{\varphi}\right\rangle,
\end{aligned}
$$

where the last term on the RHS is a high order term. Using this transport equation, one can calculate the radial profile of toroidal velocity.

\section{DISCUSSIONS AND SUMMARY}

In the last decade, the basic physics picture of transport in the plasma boundary of tokamaks and other magnetically confined fusion devices has shifted. ${ }^{113}$ This basic picture was based on the cross-field diffusive transport driven by local turbulent fluctuations, ${ }^{114}$ and in the SOL this cross-field diffusion competes with parallel classical transport toward material surfaces to establish a dynamic balance and determine the SOL radial width. ${ }^{115}$ However, in the recent years accumulating experimental evidences have shown that the crossfield transport of plasma particles, heat, and momentum is dominated by intermittent convection mediated by radially propagating filamentary structures rather than diffusion. , $, 3,10^{-}$ The presence of ballistic motion of solitary coherent objects and bursty transport events break the linear flux-gradient relationship and make the cross-field transport exhibit nonlocal character, $^{32}$ namely, transport is not determined by local parameters but is induced by propagating structures generated somewhere else.

Significant progress has been made in understanding the 2D electrostatic dynamics of blobs in the plasma boundary. ${ }^{19-25}$ Now, strong interest is arising on their 3D and electromagnetic features. ${ }^{26-29,35-37}$ This interest is enhanced by some analogies with the ELM filaments in $\mathrm{H}$-mode ${ }^{11-18}$ and the avalanchelike events in the plasma core region. ${ }^{38}$ Recently, the first experimental evidence showing the association of the propagating plasma turbulent structures with the drift-Alfvén vortices was obtained in the RFP device. ${ }^{44,45}$ Moreover, dipolar drift-Alfvén vortices were identified both in the magnetospheric cusp ${ }^{47}$ and in the magnetosheath ${ }^{48-50}$ by the four-spacecraft Cluster mission. In this contribution the generation mechanism and fundamental dynamics of drift-Alfvén vortex structures in a nonuniformly and strongly magnetized plasma are revisited. This contribution can be generally regarded as a concept upgrade from electrostatic filamentary structure ${ }^{19-25}$ to electromagnetic filamentary structure $^{26-29,35-37}$ in response to the recent experimental progress $^{43-53}$ in the context of intermittent convective transport mediated by propagating coherent structures. This is a continuation of our previous work presented in Refs. 24, 25 , 32, 34, 54, 73, and 86-89.

The main points in this paper are summarized as follows:

(A) The mesoscale structures $\left(k_{\perp} \rho_{s} \ll 1\right.$ see Table I) do not obey the standard gyrokinetic ordering of microturbulence $\left(k_{\perp} \rho_{s} \sim 1\right)$, so that a special set of ordering scheme is employed. This ordering scheme is consistent with order-unity electrostatic fluctuation levels at the plasma edge.
(B) The turbulence at fusion plasma boundary is essentially electromagnetic even for low local value of plasma $\beta$. The perpendicular dynamic timescale $\tau_{\perp}$ is comparable with the shear Alfvén time $\tau_{A}$ (see Table I), as a result the electrostatic vortex motions and the KSAWs are coupled, through the parallel current and Ampere's law, leading to field line bending.

(C) The induction part $\partial_{t} A_{\|}$of parallel electric field $E_{\|}$is of the same order as the electrostatic part $\partial_{\|} \phi$ and the parallel emf gradient $\partial_{\|} \Psi$, indicating the importance of the electromagnetic effects for mesoscale dynamics. Any imbalance in the parallel gradient force $e n \partial_{\|}(\Psi$ $-\phi$ ) on electrons will allow the parallel current $j_{\|}$and magnetic perturbations $A_{\|}$to arise, driven by the socalled nonadiabatic part of the density fluctuations, which provides a channel to exchange internal energy of particles with the magnetic energy of field line bending.

(D) The relative wave impedance $\varsigma \equiv u_{F} / u_{E}=v_{p h \|} / Z$ depends not only on the plasma $\beta$ but also on the scale $k_{\perp} \rho_{s}$. In a finite- $\beta$ plasma, for mesoscale structures $k_{\perp} \rho_{s} \ll 1, \varsigma$ is of order unity $(\varsigma<1),{ }^{92,93}$ implying that the electromagnetic effects are more important for the mesoscale dynamics than the smallscale dynamics. In the ideal MHD limit $(s=1)$, the field lines are exactly frozen in fluid elements and moving with them, and the parallel emf $\Psi$ vanishes. In the resistive MHD case $(\varsigma<1)$, only collisional resistivity can break Alfvén's frozen-in law. In progressing from the MHD limit ( $s$ $=1)$ to the kinetics limit $(s \ll 1)$, the contribution of parallel emf $\Psi$ increases and kinetic effects become alternatives to resistive diffusion for breaking Alfvén's frozen-in law and decoupling the magnetic field and plasma. The mesoscale dynamics is somewhere in between. Recent experiments on blobs from a RFP device $^{44,45}$ and ELM filaments from ASDEX Upgrade tokamak $^{46}$ suggested some MHD behaviors $s \sim 1$.

(E) In the SOL $\left(\nu^{*}>10\right.$ see Table I), plasma filaments quickly displace away from the frozen-in flux tube and drift across field lines due to the magnetic diffusion. Inside the separatrix $\left(\nu^{*}<10\right.$ see Table I), the magnetic diffusion effects are much weaker. The field lines are dragged away from the unperturbed magnetic field by the plasma filaments at a speed of $\mathbf{u}_{F}$, where $\mathbf{s}$ $\equiv u_{F} / u_{E}<1$.

(F) In a low- $\beta$ plasma the background magnetic energy is a Lagrangian invariant and $B_{0}^{2} \mathbf{u}$ is an incompressible flow. In toroidal geometry the parallel compression $\gamma_{\|}$ competes with the perpendicular compression $\gamma_{\perp}$. On the outboard midplane the parallel compression $\gamma_{\|}$ nearly vanishes, and the perpendicular compression thus dominates the density variation. At the top or bottom of the torus, the parallel and perpendicular compressions are of the same order. The spatial dependence of $\gamma_{\|} / \gamma_{\perp}$ in a torus is one of the origins of ballooning structures associated with the filamentary phenomena in toroidal geometry. 
(G) In a tokamak, when the perpendicular interchange motions and the KSAW are coupled, the KSAWs are launched from the unfavorable curvature region. The generated magnetic energy of field line bending and perpendicular kinetic energy of convective motion, or the parallel current density and the electrostatic vorticity, are related through Eqs. (18)-(20).

(H) In fusion plasmas the free energy is stored in the background pressure gradient. The generation of structures requires an effective mechanism to release the free energy and do work so that the thermal energy of particles can be converted into kinetic energy of structure motion and magnetic energy of field line bending. For a low- $\beta$ plasma the energy transfer is through the reversible pressure-volume work, which can be interpreted in terms of the fundamental thermodynamic relation and the entropy equation. When the dissipation timescale is much longer than the timescale for perpendicular convective motion $\tau_{d} \gg \tau_{\perp}$ (which is a typical case in fusion plasmas except in the far SOL, see Table I), the specific entropy is a Lagrangian invariant on the timescale of $\tau_{\perp}$. The description of the energy transfer process is closed by the conservation of total energy.

(I) The acceleration of plasma filament is induced by a force unbalance in the direction of curvature radius resulting from the plasma inertial response on the timescale of $\tau_{\perp}$, reacted to satisfy quasineutrality. A restoring force associated with the resultant force of magnetic tension does work, generating the magnetic energy of field line bending. The interchange mechanism leading to radial motion of filaments is illustrated in Fig. 6.

(J) An equivalent circuit (Fig. 7) is used to illustrate the processes in a drift-Alfvén vortex filament. The curvature and grad-B drifts function as a local current source. The currents generated by this current source flow along and across the field lines compete through their effective impedance and establish current loops. Plasma inertia plays the role of a capacitor. All dissipation processes can be represented by an equivalent resistor. The process of coupling to the KSAW is similar to that occurring in an inductor.

(K) Since plasma filaments are localized structures with finite toroidal mode number. The filamentary structures present a channel for local energy exchange between particles and magnetic field perturbations, leading to breaking of the periodic orbits of particles and the toroidal symmetry of magnetic field and resulting in the violation of the adiabatic invariance associated with the poloidal magnetic flux. This is one of the fundamental kinetic mechanisms for cross-field turbulent transport in a toroidal magnetic confinement system.

(L) The structure motions are controlled by six Lagrangian invariants, namely, magnetic flux, background magnetic energy, specific entropy, total energy, magnetic momentum, and angular momentum. The conservation of magnetic momentum is consistent with the CGL double adiabatic equations. The behaviors of toroidal angular momentum density are very similar to the particle density. Because of the radial dependence of the moment of inertia in toroidal geometry, an anomalous pinch effect emerges. This effect has recently been proposed to explain the phenomena of the so-called intrinsic/spontaneous rotation in the plasma core.

(M) The intermittent convective transports of particles, heat, and momentum across magnetic field can be interpreted in terms of the ballistic motion of these solitary filamentary structures. The mode-independent part of the curvature-driven turbulent convective pinch of particles, heat, and momentum are briefly reviewed in Sec. IX. The phenomena of profile consistency, or resilience, are generally thought to be associated with these anomalous pinch effects.

The quasilinear calculation presented in Sec. IX is only qualitative. For quantitative transport prediction one needs nonlinear electromagnetic turbulence simulations. Currently there have been several attempts in this direction, see the references in Refs. 40 and 41. Moreover, fully electromagnetic nonlinear gyrokinetic theory for edge turbulence has now come to be mature. ${ }^{83,84} \mathrm{~A}$ shift to a kinetic formulation may be required to capture the kinetic effects, such as the neoclassical flow equilibrium. Efforts dedicated to the development of such gyrokinetic models of the plasma edge are now underway. ${ }^{40,41,85}$

In summary, in this paper the ordering scheme and some fundamental aspects of filamentary structures at fusion plasma edge are reviewed. The Lagrangian-invariant-based method was systemized and extended to include the electromagnetic effects. The similarity of the electromagnetic filaments in fusion plasmas and in space plasmas suggests that it could be a universal phenomenon in plasma turbulence. The importance of such phenomenon has been widely recognized. It provides a fundamental mechanism for cross-field transport at the fusion plasma edge. ${ }^{116}$ The understanding of the plasma filamentary phenomena is rapidly evolving through the combined numerical and experimental efforts, and we expect that progress in this field will be rapid in the next several years.

\section{ACKNOWLEDGMENTS}

This work, supported by the European Communities under the contract of association between EURATOM/UKAEA and the National Natural Science Foundation of China under Grant Nos. 10605028, 10721505, and 10725523, was carried out within the framework of the European Fusion Development Agreement. The views and opinions expressed herein do not necessarily represent those of the European Commission.

\section{APPENDIX: PHYSICAL PARAMETERS AND DERIVED QUANTITIES}

See Table I. 
TABLE I. Physical parameters and derived quantities for a typical filamentary structure (using the JET tokamak parameters) in the pedestal region (in H-mode) or periphery region (in L-mode), in the vicinity of separatrix and in the SOL, assuming a pure deuterium plasma. We define the region between the pedestal (or periphery) and the separatrix as the plasma edge, and the region outside the separatrix as the SOL. See text for explanation and discussion.

\begin{tabular}{|c|c|c|c|}
\hline Quantity, symbol (unit) & Pedestal or periphery & Separatrix & SOL \\
\hline Particle density, $n\left(\mathrm{~m}^{-3}\right)$ & $1 \times 10^{20}$ & $3 \times 10^{19}$ & $5 \times 10^{18}$ \\
\hline Temperature, $T_{e}, T_{i}(\mathrm{eV})$ & $T_{e}=T_{i}=1000$ & $T_{e}=T_{i}=100$ & $T_{e}=T_{i} / 2=10$ \\
\hline Magnetic field, $B(\mathrm{~T})$ & 3 & 3 & 3 \\
\hline Major radius, $R(\mathrm{~m})$ & 3 & 3 & 3 \\
\hline Safety factor, $q$ & 3 & 4 & 5 \\
\hline Parallel length, $L_{\|} \sim \pi q R$ (m) & 30 & 40 & 30 \\
\hline Background pressure gradient length, $L_{p}(\mathrm{~mm})$ & $\sim 50$ & $\sim 50$ & $\sim 100$ \\
\hline Typical vortex width, $L_{\perp}(\mathrm{mm})$ & $\sim 10$ & $\sim 10$ & $\sim 10$ \\
\hline Transverse motion velocity, $u_{\perp}(\mathrm{km} / \mathrm{s})$ & $\sim 1$ & $\sim 1$ & $\sim 1$ \\
\hline Experimental cross-field diffusivity, $D_{\text {eff }}\left(\mathrm{m}^{2} / \mathrm{s}\right)$ & $\sim 0.1$ & $\sim 1$ & $\sim 2$ \\
\hline Vortex lifetime or eruption time, $\tau_{\text {life }}(\mu \mathrm{s})$ & $\sim 100$ & $\sim 100$ & $\sim 100$ \\
\hline Ion gyrofrequency, $f_{c i}=e B / 2 \pi m_{i}(\mathrm{MHz})$ & 23 & 23 & 23 \\
\hline Ion plasma frequency, $f_{p i}=\omega_{p i} / 2 \pi(\mathrm{MHz})$ & $1.5 \times 10^{3}$ & 810 & 330 \\
\hline Debye length, $\lambda_{D}=\left(\varepsilon_{0} T_{e} / n e^{2}\right)^{1 / 2}(\mathrm{~mm})$ & $2.4 \times 10^{-2}$ & $1.4 \times 10^{-2}$ & $1 \times 10^{-2}$ \\
\hline Ion gyroradius, $\rho_{i}=v_{t h i} / \omega_{c i}(\mathrm{~mm})$ & 2.2 & 0.7 & 0.3 \\
\hline Ion poloidal gyroradius, $\rho_{\theta i}=\rho_{i} B / B_{\theta}(\mathrm{mm})$ & 43 & 13.6 & 6.1 \\
\hline Ion sound gyroradius, $\rho_{s}=c_{s} / \omega_{c i}(\mathrm{~mm})$ & 2.2 & 0.7 & 0.26 \\
\hline Quasineutrality, $\rho_{i} / \lambda_{D} \sim c / v_{A}=\omega_{p i} / \omega_{c i}$ & 92 & 50 & 29 \\
\hline Magnetic curvature, $\rho_{i} / R$ & $7 \times 10^{-4}$ & $2.3 \times 10^{-4}$ & $1 \times 10^{-4}$ \\
\hline Magnetization parameter, $\delta=\rho_{s} / L_{\perp}$ & 0.22 & 0.07 & 0.026 \\
\hline Magnetic diffusion length, $L_{m}=\left(\tau_{\perp} D_{m}\right)^{1 / 2}(\mathrm{~mm})$ & 0.6 & 3.3 & 17 \\
\hline Electron inertial length, $L_{p e}=c / \omega_{p e}(\mathrm{~mm})$ & 0.5 & 1 & 2.4 \\
\hline Ion inertial length, $L_{p i}=c / \omega_{p i}(\mathrm{~mm})$ & 32 & 59 & 144 \\
\hline Electron mean free path, $\lambda_{e}=v_{t h e} \tau_{e i}(\mathrm{~m})$ & 140 & 5 & 0.35 \\
\hline Ion mean free path, $\lambda_{i}=v_{t h i} \tau_{i i}(\mathrm{~m})$ & 170 & 7 & 2 \\
\hline Collisionality, $\nu^{*}=L_{\|} / \lambda_{e}$ & 0.2 & 7.7 & 86 \\
\hline Spatial anisotropy, $L_{\perp} / L_{\|} \sim \delta \beta_{e}^{1 / 2}$ & $3.3 \times 10^{-4}$ & $2.5 \times 10^{-4}$ & $3.3 \times 10^{-4}$ \\
\hline Specific pressure, $\beta_{e}=2 \mu_{0} p_{e} / B^{2} \sim\left(c_{s} / v_{A}\right)^{2}=\left(\rho_{s} / L_{p i}\right)^{2}$ & $4.5 \times 10^{-3}$ & $1.3 \times 10^{-4}$ & $2.2 \times 10^{-6}$ \\
\hline Mass ratio for deuterium, $m_{e} / m_{i}$ & $2.7 \times 10^{-4}$ & $2.7 \times 10^{-4}$ & $2.7 \times 10^{-4}$ \\
\hline Alfvén speed, $v_{A}=\left(B^{2} / \mu_{0} n m_{i}\right)^{1 / 2}(\mathrm{~km} / \mathrm{s})$ & $4.6 \times 10^{3}$ & $8.5 \times 10^{3}$ & $2 \times 10^{4}$ \\
\hline Electron thermal speed, $v_{\text {the }}=\left(2 T_{e} / m_{e}\right)^{1 / 2}(\mathrm{~km} / \mathrm{s})$ & $2 \times 10^{4}$ & $6 \times 10^{3}$ & $2 \times 10^{3}$ \\
\hline Ion thermal speed, $v_{t h i}=\left(2 T_{i} / m_{i}\right)^{1 / 2}(\mathrm{~km} / \mathrm{s})$ & 310 & 98 & 44 \\
\hline Ion sound speed, $c_{s} \sim \beta^{1 / 2} v_{A}(\mathrm{~km} / \mathrm{s})$ & 310 & 98 & 38 \\
\hline Transverse Mach number, $M=u_{\perp} / c_{s}$ & 0.003 & 0.01 & 0.03 \\
\hline Interchange velocity, $u_{\mathrm{int}}=c_{S}\left(L_{\perp} / R\right)^{1 / 2}(\mathrm{~km} / \mathrm{s})$ & 18 & 6 & 2 \\
\hline Froude number, $\mathrm{Fr}=u_{\perp} / u_{\text {int }}$ & 0.06 & 0.2 & 0.5 \\
\hline Magnetic diffusivity, $D_{m}=\eta / \mu_{0}=L_{p e}^{2} / \tau_{e i}\left(\mathrm{~m}^{2} / \mathrm{s}\right)$ & 0.04 & 1 & 30 \\
\hline Classical cross-field diffusivity, $D_{c l}=\beta_{e} D_{m}\left(\mathrm{~m}^{2} / \mathrm{s}\right)$ & $1.7 \times 10^{-4}$ & $1.4 \times 10^{-4}$ & $6.8 \times 10^{-5}$ \\
\hline Bohm diffusivity, $D_{B}=T_{e} / 16 B\left(\mathrm{~m}^{2} / \mathrm{s}\right)$ & 20 & 2 & 0.2 \\
\hline Kinematic viscosity, $\nu=\rho_{i}^{2} / \tau_{i i}\left(\mathrm{~m}^{2} / \mathrm{s}\right)$ & $8.4 \times 10^{-3}$ & $6.6 \times 10^{-3}$ & $2.2 \times 10^{-3}$ \\
\hline Electron thermal conductivity, $\chi_{\|}^{e}=3.2 \tau_{e i} T_{e} / m_{e}\left(\mathrm{~m}^{2} / \mathrm{s}\right)$ & $4 \times 10^{9}$ & $5 \times 10^{7}$ & $1 \times 10^{6}$ \\
\hline Ion thermal conductivity, $\chi_{\|}^{i}=3.9 \tau_{i i} T_{i} / m_{i}\left(\mathrm{~m}^{2} / \mathrm{s}\right)$ & $1 \times 10^{8}$ & $1.3 \times 10^{6}$ & $1.6 \times 10^{5}$ \\
\hline Vortex turnover time, $\tau_{\perp}=L_{\perp} / u_{\perp}(\mu \mathrm{s})$ & $\sim 10$ & $\sim 10$ & $\sim 10$ \\
\hline Shear Alfvén time, $\tau_{A}=L_{\|} / v_{A}(\mu \mathrm{s})$ & 6.5 & 4.7 & 1.4 \\
\hline Compressional Alfvén time, $\tau_{A \perp}=L_{\perp} / v_{A}(\mu \mathrm{s})$ & $2 \times 10^{-3}$ & $1 \times 10^{-3}$ & $5 \times 10^{-4}$ \\
\hline Parallel convective time, $\tau_{\|}=L_{\|} / c_{s}(\mu \mathrm{s})$ & 100 & 410 & 790 \\
\hline Perpendicular sound transit time, $\tau_{s}=L_{\perp} / c_{s}(\mu \mathrm{s})$ & 0.03 & 0.1 & 0.26 \\
\hline Ion transit time, $\tau_{i \|}=L_{\|} / v_{t h i}(\mu \mathrm{s})$ & 100 & 410 & 690 \\
\hline Electron transit time, $\tau_{e \|}=L_{\|} / v_{\text {the }}(\mu \mathrm{s})$ & 1.6 & 6.7 & 16 \\
\hline Magnetic diffusion time, $\tau_{m}=L_{\perp}^{2} / D_{m}(\mu \mathrm{s})$ & 2600 & 93 & 3 \\
\hline Magnetic reconnection time, $\tau_{K}=\left(\tau_{m} \tau_{A}\right)^{1 / 2}(\mu \mathrm{s})$ & 130 & 20 & 2 \\
\hline Viscous dissipation time, $\tau_{\nu}=L_{\perp}^{2} / \nu(\mu \mathrm{s})$ & $1.2 \times 10^{4}$ & $1.5 \times 10^{4}$ & $4.6 \times 10^{4}$ \\
\hline
\end{tabular}


TABLE I. (Continued.)

\begin{tabular}{lccc}
\hline \hline Quantity, symbol (unit) & Pedestal or periphery & Separatrix & SOL \\
\hline Electron thermal conductive time, $\tau_{\|}^{e}=L_{\|}^{2} / \chi_{\|}^{e}(\mu \mathrm{s})$ & 0.2 & 32 & 860 \\
Ion thermal conductive time, $\tau_{\|}^{i}=L_{\|}^{2} / \chi_{\|}^{i}(\mu \mathrm{s})$ & 9 & $1.2 \times 10^{3}$ & $5.7 \times 10^{3}$ \\
Electron-ion collisional time, $\tau_{e i} \propto T_{e}^{3 / 2} / n(\mu \mathrm{s})$ & 7 & 0.9 & 0.2 \\
Ion-ion collisional time, $\tau_{i i} \propto T_{i}^{3 / 2} / n(\mu \mathrm{s})$ & 550 & 70 & 42 \\
Electron-ion energy exchange time, $\tau_{e x}=\tau_{e i} m_{i} / 3 m_{e}(\mu \mathrm{s})$ & 9000 & 1100 & 230 \\
Electron temperature isotropization, $\tau_{T e}(\mu \mathrm{s})$ & 26 & 3 & 0.7 \\
Ion temperature isotropization, $\tau_{T i}(\mu \mathrm{s})$ & 1400 & 180 & 100 \\
Interchange acceleration time, $\tau_{\text {int }}=\left(R L_{\perp}\right)^{1 / 2} / c_{s}(\mu \mathrm{s})$ & 0.6 & 2 & 4.6 \\
Magnetic Reynolds number, $R_{m}=u_{\perp} L_{\perp} / D_{m}$ & 260 & 9.3 & 0.3 \\
Lundquist number, $S_{m}=R_{m} v_{A} / u_{\perp}$ & $1.2 \times 10^{6}$ & $8 \times 10^{4}$ & $7 \times 10^{3}$ \\
Magnetic Prandtl number, $P_{m}=R_{m} / \mathrm{Re}=\nu / D_{m}$ & 0.2 & $6 \times 10^{-3}$ & $7 \times 10^{-5}$ \\
Reynolds number, Re $=u_{\perp} L_{\perp} / \nu$ & $1.2 \times 10^{3}$ & $1.5 \times 10^{3}$ & $4.6 \times 10^{3}$ \\
Schmidt number, Sc $=\nu / D_{c l}$ & 49 & 46 & 32 \\
Péclet number, Pe $=\operatorname{Re} \mathrm{Sc}=u_{\perp} L_{\perp} / D_{c l}$ & $6 \times 10^{4}$ & $7 \times 10^{4}$ & $1.5 \times 10^{5}$ \\
Interchange acceleration, $g=c_{s}^{2} / R\left(\mathrm{~m} / \mathrm{s}^{2}\right)$ & $3 \times 10^{10}$ & $3 \times 10^{9}$ & $5 \times 10^{8}$ \\
Rayleigh number, Ra $=g L_{\perp}^{3} / \nu^{2}$ & $4.5 \times 10^{8}$ & $7.3 \times 10^{7}$ & $1 \times 10^{8}$ \\
Normalized blob size, $a_{*}=\rho_{s}\left(L_{\|}^{2} / R \rho_{s}\right)^{1 / 5}(\mathrm{~mm})$ & 23 & 10 & 4.3 \\
Normalized blob velocity, $v_{*}=c_{s}\left(a_{*} / R\right)^{1 / 2}(\mathrm{~km} / \mathrm{s})$ & 27 & 5.7 & 1.4 \\
Inertial parameter, $\hat{\mathrm{\omega}}=\left(m_{e} / m_{i}\right)\left(q R / L_{p}\right)^{2}$ & 8.8 & 15.7 & 24.5 \\
Inductive parameter, $\hat{\beta}=\beta_{e}\left(q R / L_{p}\right)^{2}$ & 145 & 7.7 & 0.2 \\
Resistive parameter, $C=0.51 \hat{\mathrm{\omega}} L_{p} / c_{s} \tau_{e i}$ & 0.1 & 4.7 & 89 \\
\hline \hline
\end{tabular}

${ }^{1}$ B. A. Carreras, IEEE Trans. Plasma Sci. 25, 1281 (1997).

${ }^{2}$ S. J. Zweben, J. A. Boedo, O. Grulke, C. Hidalgo, B. LaBombard, R. J. Maqueda, P. Scarin, and J. L. Terry, Plasma Phys. Controlled Fusion 49, S1 (2007).

${ }^{3}$ J. A. Boedo, J. Nucl. Mater. 390-391, 29 (2009).

${ }^{4}$ O. Grulke, T. Klinger, M. Endler, A. Piel, and the W7-AS Team, Phys. Plasmas 8, 5171 (2001).

${ }^{5}$ M. Spolaore, V. Antoni, E. Spada, H. Bergsåker, R. Cavazzana, J. R. Drake, E. Martines, G. Regnoli, G. Serianni, and N. Vianello, Phys. Rev. Lett. 93, 215003 (2004).

${ }^{6}$ N. Ben Ayed, A. Kirk, B. Dudson, S. Tallents, R. G. L. Vann, H. R. Wilson, and the MAST team, Plasma Phys. Controlled Fusion 51, 035016 (2009).

${ }^{7}$ I. Furno, B. Labit, M. Podestà, A. Fasoli, S. H. Müller, F. M. Poli, P. Ricci, C. Theiler, S. Brunner, A. Diallo, and J. Graves, Phys. Rev. Lett. 100, 055004 (2008)

${ }^{8}$ T. A. Carter, Phys. Plasmas 13, 010701 (2006).

${ }^{9}$ W. Horton and Y.-H. Ichikawa, Chaos and Structures in Nonlinear Plasmas (World Scientific, Singapore, 1996).

${ }^{10}$ G. Serianni, M. Agostini, V. Antoni, R. Cavazzana, E. Martines, F. Sattin, P. Scarin, E. Spada, M. Spolaore, N. Vianello, and M. Zuin, Plasma Phys. Controlled Fusion 49, B267 (2007).

${ }^{11}$ W. Fundamenski, W. Sailer, and JET EFDA contributors, Plasma Phys. Controlled Fusion 46, 233 (2004).

${ }^{12}$ M. Endler, I. García-Cortés, C. Hidalgo, G. F. Matthews, ASDEX Team, and JET Team, Plasma Phys. Controlled Fusion 47, 219 (2005).

${ }^{13}$ J. A. Boedo, D. L. Rudakov, E. Hollmann, D. S. Gray, K. H. Burrell, R. A. Moyer, G. R. McKee, R. Fonck,P. C. Stangeby, T. E. Evans, P. B. Snyder, A. W. Leonard, M. A. Mahdavi, M. J. Schaffer, W. P. West, M. E. Fenstermacher, M. Groth, S. L. Allen, C. Lasnier, G. D. Porter, N. S. Wolf, R. J. Colchin, L. Zeng, G. Wang, J. G. Watkins, T. Takahashi, and DIII-D Team, Phys. Plasmas 12, 072516 (2005).

${ }^{14}$ A. Kirk, N. Ben Ayed, G. Counsell, B. Dudson, T. Eich, A. Herrmann, B. Koch, R. Martin, A. Meakins, S. Saarelma, R. Scannell, S. Tallents, M. Walsh, H. R. Wilson, and the MAST Team, Plasma Phys. Controlled Fusion 48, B433 (2006).

${ }^{15}$ A. Herrmann, A. Kirk, A. Schmid, B. Koch, M. Laux, M. Maraschek, H. W. Mueller, J. Neuhauser, V. Rohde, M. Tsalas, E. Wolfrum, and the ASDEX Upgrade Team, J. Nucl. Mater. 363-365, 528 (2007).
${ }^{16}$ A. Schmid, A. Herrmann, H. W. Mueller, and the ASDEX Upgrade Team, Plasma Phys. Controlled Fusion 50, 045007 (2008).

${ }^{17}$ B. D. Dudson, N. Ben Ayed, A. Kirk, H. R. Wilson, G. Counsell, X. Xu, M. Umansky, P. B. Snyder, B. Lloyd, and the MAST Team, Plasma Phys. Controlled Fusion 50, 124012 (2008).

${ }^{18}$ C. Silva, W. Fundamenski, A. Alonso, B. Gonçalves, C. Hidalgo, M. A. Pedrosa, R. A. Pitts, M. Stemp, and JET EFDA Contributors, Plasma Phys. Controlled Fusion 51, 105001 (2009).

${ }^{19}$ S. I. Krasheninnikov, Phys. Lett. A 283, 368 (2001).

${ }^{20}$ D. A. D’Ippolito, J. R. Myra, and S. I. Krasheninnikov, Phys. Plasmas 9, 222 (2002).

${ }^{21}$ N. Bian, S. Benkadda, J.-V. Paulsen, and O. E. Garcia, Phys. Plasmas 10, 671 (2003)

${ }^{22}$ O. E. Garcia, V. Naulin, A. H. Nielsen, and J. Juul Rasmussen, Phys. Rev. Lett. 92, 165003 (2004).

${ }^{23}$ A. Y. Aydemir, Phys. Plasmas 12, 062503 (2005).

${ }^{24}$ O. E. Garcia, N. H. Bian, V. Naulin, A. H. Nielsen, and J. J. Rasmussen, Phys. Plasmas 12, 090701 (2005)

${ }^{25}$ O. E. Garcia, N. H. Bian, and W. Fundamenski, Phys. Plasmas 13, 082309 (2006).

${ }^{26}$ S. I. Krasheninnikov, D. D. Ryutov, and G. Yu, J. Plasma Fusion Res. 6, 139 (2004)

${ }^{27}$ J. R. Myra and D. A. D’Ippolito, Phys. Plasmas 12, 092511 (2005).

${ }^{28}$ D. D. Ryutov, Phys. Plasmas 13, 122307 (2006).

${ }^{29}$ J. R. Myra, Phys. Plasmas 14, 102314 (2007).

${ }^{30}$ J. R. Myra, D. A. Russell, and D. A. D'Ippolito, Phys. Plasmas 15, 032304 (2008)

${ }^{31}$ S. Ishiguro and H. Hasegawa, J. Plasma Phys. 72, 1233 (2006).

${ }^{32}$ V. Naulin, J. Nucl. Mater. 363-365, 24 (2007).

${ }^{33}$ S. I. Krasheninnikov, D. A. D'Ippolito, and J. R. Myra, J. Plasma Phys. 74, 679 (2008).

${ }^{34}$ W. Fundamenski, O. E. Garcia, V. Naulin, R. A. Pitts, A. H. Nielsen, J. Juul Rasmussen, J. Horacek, J. P. Graves, and JET EFDA Contributors, Nucl. Fusion 47, 417 (2007).

${ }^{35}$ V. Naulin, Phys. Plasmas 10, 4016 (2003).

${ }^{36}$ B. D. Scott, Plasma Phys. Controlled Fusion 49, S25 (2007).

${ }^{37}$ T. T. Ribeiro and B. Scott, Plasma Phys. Controlled Fusion 50, 055007 (2008)

${ }^{38}$ P. A. Politzer, Phys. Rev. Lett. 84, 1192 (2000). 
${ }^{39}$ Y. Hamada, T. Watari, A. Nishizawa, K. Narihara, Y. Kawasumi, T. Ido, M. Kojima, and K. Toi, Phys. Rev. Lett. 96, 115003 (2006).

${ }^{40}$ D. A. Batchelor, M. Beck, A. Becoulet, R. V. Budny, C. S. Chang, P. H. Diamond, J. Q. Dong, G. Y. Fu, A. Fukuyama, T. S. Hahm, D. E. Keyes, Y. Kishimoto, S. Klasky, L. L. Lao, K. Li, Z. Lin, B. Ludaescher, J. Manickam, N. Nakajima, T. Ozeki, N. Podhorszki, W. M. Tang, M. A. Vouk, R. E. Waltz, S. J. Wang, H. R. Wilson, X. Q. Xu, M. Yagi, and F. Zonca, Plasma Sci. Technol. 9, 312 (2007).

${ }^{41}$ G. L. Falchetto, B. D. Scott, P. Angelino, A. Bottino, T. Dannert, V. Grandgirard, S. Janhunen, F. Jenko, S. Jolliet, A. Kendl, B. F. McMillan, V. Naulin, A. H. Nielsen, M. Ottaviani, A. G. Peeters, M. J. Pueschel, D. Reiser, T. T. Ribeiro, and M. Romanelli, Plasma Phys. Controlled Fusion 50, 124015 (2008).

${ }^{42}$ F. L. Hinton, M. N. Rosenbluth, and R. E. Waltz, Phys. Plasmas 10, 168 (2003).

${ }^{43}$ O. Grulke, S. Ullrich, T. Windisch, and T. Klinger, Plasma Phys. Controlled Fusion 49, B247 (2007).

${ }^{44}$ M. Spolaore, N. Vianello, M. Agostini, R. Cavazzana, E. Martines, P. Scarin, G. Serianni, E. Spada, M. Zuin, and V. Antoni, Phys. Rev. Lett. 102, 165001 (2009).

${ }^{45}$ N. Vianello, M. Spolaore, E. Martines, R. Cavazzana, G. Serianni, M. Zuin, E. Spada, and V. Antoni, "Drift-Alfvén vortex structures in the edge region of a fusion relevant plasma," Phys. Rev. Lett. (submitted).

${ }^{46}$ E. Martines, N. Vianello, D. Sundkvist, M. Spolaore, M. Zuin, M. Agostini, V. Antoni, R. Cavazzana, C. Ionita, M. Maraschek, F. Mehlmann, H. W. Müller, V. Naulin, J. J. Rasmussen, V. Rohde, P. Scarin, R. Schrittwieser, G. Serianni, E. Spada, the RFX-mod Team, and the ASDEX Upgrade Team, Plasma Phys. Controlled Fusion 51, 124053 (2009); N. Vianello, V. Naulin, R. Schrittwieser, H. W. Muller, M. Zuin, C. Ionita, J. J. Rasmussen, F. Mehlmann, V. Rohde, R. Cavazzana, M. Maraschek, and the ASDEX Upgrade Team, "Direct observation of current in type I ELM filaments on Asdex Upgrade," Phys. Rev. Lett. (submitted).

${ }^{47}$ M. L. Goldstein, Nature (London) 436, 782 (2005).

${ }^{48}$ D. Sundkvist, V. Krasnoselskikh, P. K. Shukla, A. Vaivads, M. Andre, S. Buchert, and H. Reme, Nature (London) 436, 825 (2005).

${ }^{49}$ O. Alexandrova, A. Mangeney, M. Maksimovic, N. Cornilleau-Wehrlin, J. M. Bosqued, and M. André, J. Geophys. Res. 111, A12208, doi:10.1029/ 2006JA011934 (2006).

${ }^{50}$ O. Alexandrova, Nonlinear Processes in Geophysics 15, 95 (2008).

${ }^{51}$ D. Sundkvist and S. D. Bale, Phys. Rev. Lett. 101, 065001 (2008).

${ }^{52}$ O. Alexandrova and J. Saur, Geophys. Res. Lett. 35, L15102, doi:10.1029/2008GL034411 (2008).

${ }^{53}$ S. Tomczyk, S. W. McIntosh, S. L. Keil, P. G. Judge, T. Schad, D. H. Seeley, and J. Edmondson, Science 317, 1192 (2007).

${ }^{54}$ W. Fundamenski, V. Naulin, T. Neukirch, O. E. Garcia, and J. Juul Rasmussen, Plasma Phys. Controlled Fusion 49, R43 (2007).

${ }^{55}$ J. E. Wahlund, P. Louarn, T. Chust, H. de Feraudy, A. Roux, B. Holback, P. O. Dovner, and G. Holmgren, Geophys. Res. Lett. 21, 1831, doi:10.1029/94GL01289 (1994).

${ }^{56}$ A. A. Pimenta, Y. Sahai, J. A. Bittencourt, and F. J. Rich, Geophys. Res. Lett. 34, L02820, doi:10.1029/2006GL028529 (2007).

${ }^{57}$ A. Loarte, B. Lipschultz, A. S. Kukushkin, G. F. Matthews, P. C. Stangeby, N. Asakura, G. F. Counsell, G. Federici, A. Kallenbach, K. Krieger, A. Mahdavi, V. Philipps, D. Reiter, J. Roth, J. Strachan, D. Whyte, R. Doerner, T. Eich, W. Fundamenski, A. Herrmann, M. Fenstermacher, P. Gendrih, M. Groth, A. Kirschner, S. Konoshima, B. LaBombard, P. Lang, A. W. Leonard, P. Monier-Garbet, R. Neu, H. Pacher, B. Pegourie, R. A. Pitts, S. Takamura, J. Terry, E. Tsitrone, and the ITPA Scrape-off Layer and Divertor Physics Topical Group, Nucl. Fusion 47, S203 (2007).

${ }^{58}$ J. P. Gunn, C. Boucher, M. Dionne, I. Duran, V. Fuchs, T. Loarer, I. Nanobashvili, R. Pánek, J.-Y. Pascal, F. Saint-Laurent, J. Stöckel, T. Van Rompuy, R. Zagórski, J. Adámek, J. Bucalossi, R. Dejarnac, P. Devynck, P. Hertout, M. Hron, G. Lebrun, P. Moreau, F. Rimini, A. Sarkissian, and G. Van Oost, J. Nucl. Mater. 363-365, 484 (2007).

${ }^{59}$ N. Smick, B. LaBombard, and C. S. Pitcher, J. Nucl. Mater. 337-339, 281 (2005).

${ }^{60}$ S. K. Erents, R. A. Pitts, W. Fundamenski, J. P. Gunn, and G. F. Matthews, Plasma Phys. Controlled Fusion 46, 1757 (2004).

${ }^{61}$ N. Asakura and ITPA SOL and Divertor Topical Group, J. Nucl. Mater. 363-365, 41 (2007).

${ }^{62}$ V. V. Yankov, JETP Lett. 60, 171 (1994).

${ }^{63}$ J. Nycander and V. V. Yankov, Phys. Plasmas 2, 2874 (1995).
${ }^{64}$ M. B. Isichenko, A. V. Gruzinov, and P. H. Diamond, Phys. Rev. Lett. 74, 4436 (1995).

${ }^{65}$ M. B. Isichenko and N. V. Petviashvili, Phys. Plasmas 2, 3650 (1995).

${ }^{66}$ M. B. Isichenko, A. V. Gruzinov, P. H. Diamond, and P. N. Yushmanov, Phys. Plasmas 3, 1916 (1996).

${ }^{67}$ J. Nycander and V. V. Yankov, Phys. Scr. T63, 174 (1996).

${ }^{68}$ V. V. Yankov and J. Nycander, Phys. Plasmas 4, 2907 (1997).

${ }^{69}$ M. B. Isichenko and V. V. Yankov, Phys. Rep. 283, 161 (1997).

${ }^{70}$ J. Nycander and J. J. Rasmussen, Plasma Phys. Controlled Fusion 39, 1861 (1997).

${ }^{71}$ V. Naulin, J. Nycander, and J. J. Rasmussen, Phys. Rev. Lett. 81, 4148 (1998).

${ }^{72}$ X. Garbet, N. Dubuit, E. Asp, Y. Sarazin, C. Bourdelle, P. Ghendrih, and G. T. Hoang, Phys. Plasmas 12, 082511 (2005).

${ }^{73}$ O. E. Garcia, N. H. Bian, V. Naulin, A. H. Nielsen, and J. Juul Rasmussen, Phys. Scr. T122, 104 (2006).

${ }^{74}$ J. Harvey, Phys. Today 48, 32 (1995).

${ }^{75}$ E. Fermi, Thermodynamics (Prentice-Hall, New York, 1937).

${ }^{76}$ Ö. D. Gürcan, P. H. Diamond, and T. S. Hahm, Phys. Rev. Lett. 100, 135001 (2008)

${ }^{77}$ T. S. Hahm, P. H. Diamond, O. D. Gurcan, and G. Rewoldt, Phys. Plasmas 15, 055902 (2008).

${ }^{78}$ F. Wagner and U. Stroth, Plasma Phys. Controlled Fusion 35, 1321 (1993).

${ }^{79}$ G. S. Xu, V. Naulin, W. Fundamenski, C. Hidalgo, J. A. Alonso, C. Silva, B. Gonçalves, A. H. Nielsen, J. J. Rasmussen, S. I. Krasheninnikov, B. N. Wan, and M. Stamp, Nucl. Fusion 49, 092002 (2009).

${ }^{80}$ B. D. Scott, Plasma Phys. Controlled. Fusion 39, 1635 (1997).

${ }^{81}$ B. D. Scott, Plasma Phys. Controlled. Fusion 45, A385 (2003).

${ }^{82}$ B. D. Scott, Plasma Phys. Controlled. Fusion 48, B277 (2006).

${ }^{83}$ H. Qin, R. H. Cohen, W. M. Nevins, and X. Q. Xu, Phys. Plasmas 14, 056110 (2007).

${ }^{84}$ T. S. Hahm, L. Wang, and J. Madsen, Phys. Plasmas 16, 022305 (2009).

${ }^{85}$ R. H. Cohen and X. Q. Xu, Contrib. Plasma Phys. 48, 212 (2008).

${ }^{86}$ O. E. Garcia, V. Naulin, A. H. Nielsen, and J. J. Rasmussen, Phys. Plasmas 12, 062309 (2005).

${ }^{87}$ O. E. Garcia, V. Naulin, A. H. Nielsen, and J. J. Rasmussen, Phys. Scr. T122, 89 (2006).

${ }^{88}$ O. E. Garcia, J. Horacek, R. A. Pitts, A. H. Nielsen, W. Fundamenski, J. P. Graves, V. Naulin, and J. J. Rasmussen, Plasma Phys. Controlled Fusion 48, L1 (2006).

${ }^{89}$ O. E. Garcia, J. Plasma Fusion Res. 4, 019 (2009).

${ }^{90}$ Here we use the term "charge polarization" instead of "charge separation," since in a quasineutral plasma the net charge is negligible, $\sigma=\sigma_{b}+\sigma_{f}$ $=\varepsilon_{0} \nabla \cdot \mathbf{E}$, where the net charge $\sigma$ is composed of the bound charge $\sigma_{b}=$ $-\nabla \cdot \mathbf{P}_{b}$ and the free charge $\sigma_{f}=\nabla \cdot \mathbf{D}$. Here, $\mathbf{P}_{b}$ is the polarization per unit volume and $\mathbf{D}=\mathbf{P}_{b}+\varepsilon_{0} \mathbf{E} \approx \mathbf{P}_{b}$ is the electric displacement field. The conservation of charge requires $\partial_{t} \sigma+\nabla \cdot \mathbf{j}=\partial_{t} \sigma_{b}+\nabla \cdot \mathbf{j}_{p}=\partial_{t} \sigma_{f}+\nabla \cdot \mathbf{j}_{f}=0$, where the plasma current $\mathbf{j}=\mathbf{j}_{f}+\mathbf{j}_{p}+\mathbf{j}_{b}$ is composed of the free current $\mathbf{j}_{f}$, the polarization current $\mathbf{j}_{p}$, and the magnetization (bound) current $\mathbf{j}_{b}$. Noting $\nabla \cdot \mathbf{j}_{b}=0$. The details about these current components are addressed in Sec. VI.

${ }^{91}$ J. J. Ramos, Phys. Plasmas 14, 052506 (2007).

${ }^{92}$ O. G. Onishchenko, V. V. Krasnoselskikh, and O. A. Pokhotelov, Phys. Plasmas 15, 022903 (2008).

${ }^{93}$ O. G. Onishchenko, O. A. Pokhotelov, V. V. Krasnoselskikh, and S. I. Shatalov, Ann. Geophys. 27, 639 (2009).

${ }^{94}$ A. B. Mikhailovskii, V. P. Lakhin, G. D. Aburdzhaniya, L. A. Mikhailovskaya, O. G. Onishchenko, and A. I. Smolyakov, Plasma Phys. Controlled. Fusion 29, 001 (1987).

${ }^{95}$ V. I. Petviashvili and O. A. Pokhotelov, Solitary Waves in Plasmas and in the Atmosphere (Gordon \& Breach, London, 1992).

${ }^{96}$ V. I. Petviashvili, Plasma Phys. Rep. 19, 256 (1993).

${ }^{97}$ P. K. Shukla, M. Y. Yu, and R. K. Varma, Phys. Fluids 28, 1719 (1985).

${ }^{98}$ W. Horton, Rev. Mod. Phys. 71, 735 (1999).

${ }^{99}$ P. B. Synder, Ph.D. thesis, Princeton University, 1999.

${ }^{100}$ D. Biskamp, Nonlinear Magnetohydrodynamics (Cambridge University Press, Cambridge, 1993).

${ }^{101}$ G. Fuhr, P. Beyer, S. Benkadda, and X. Garbet, Phys. Rev. Lett. 101, 195001 (2008).

${ }^{102}$ S. I. Braginskii, in Reviews of Plasma Physics, edited by M. A. Leontovich (Consultants Bureau, New York, 1965), Vol. I, p. 205.

${ }^{103}$ O. E. Garcia, Eur. J. Phys. 24, 331 (2003). 
${ }^{104}$ H. Qin, W. M. Tang, G. Rewoldt, and W. W. Lee, Phys. Plasmas 7, 991 (2000).

${ }^{105}$ G. Q. Yu, S. I. Krasheninnikov, and P. N. Guzdar, Phys. Plasmas 13, 042508 (2006).

${ }^{106}$ J. F. Drake and T. M. Antonsen, Phys. Fluids 27, 898 (1984).

${ }^{107}$ J. R. Myra, D. A. Russell, and D. A. D'Ippolito, Phys. Plasmas 13, 112502 (2006).

${ }^{108}$ G. L. Chew, M. L. Goldberger, and F. E. Low, Proc. R. Soc. London, Ser. A 236, 112 (1956).

${ }^{109}$ F. L. Hinton and S. K. Wong, Phys. Fluids 28, 3082 (1985).

${ }^{110}$ J. E. Rice, A. Ince-Cushman, J. S. deGrassie, L.-G. Eriksson, Y. Sakamoto, A. Scarabosio, A. Bortolon, K. H. Burrell, B. P. Duval, C. FenziBonizec, M. J. Greenwald, R. J. Groebner, G. T. Hoang, Y. Koide, E. S. Marmar, A. Pochelon, and Y. Podpaly, Nucl. Fusion 47, 1618 (2007).

${ }^{111}$ T. S. Hahm, P. H. Diamond, O. D. Gurcan, and G. Rewoldt, Phys. Plasmas 14, 072302 (2007).
${ }^{112}$ N. H. Bian and O. E. Garcia, Phys. Plasmas 12, 042307 (2005).

${ }^{113}$ B. A. Carreras, J. Nucl. Mater. 337-339, 315 (2005).

${ }^{114}$ P. C. Liewer, Nucl. Fusion 25, 1281 (1985).

${ }^{115}$ P. C. Stangeby, The Plasma Boundary of Magnetic Fusion Devices (Institute of Physics, Bristol, 2000).

${ }^{116}$ E. J. Doyle, W. A. Houlberg, Y. Kamada, V. Mukhovatov, T. H. Osborne, A. Polevoi, G. Bateman, J. W. Connor, J. G. Cordey, T. Fujita, X. Garbet, T. S. Hahm, L. D. Horton, A. E. Hubbard, F. Imbeaux, F. Jenko, J. E. Kinsey, Y. Kishimoto, J. Li, T. C. Luce, Y. Martin, M. Ossipenko, V. Parai, A. Peeters, T. L. Rhodes, J. E. Rice, C. M. Roach, V. Rozhansky, F. Ryter, G. Saibene, R. Sartori, A. C. C. Sips, J. A. Snipes, M. Sugihara, E. J. Synakowski, H. Takenaga, T. Takizuka, K. Thomsen, M. R. Wade, H. R. Wilson, ITPA Transport Physics Topical Group, ITPA Confinement Database and Modelling Topical Group, and ITPA Pedestal and Edge Topical Group, Nucl. Fusion 47, S18 (2007). 